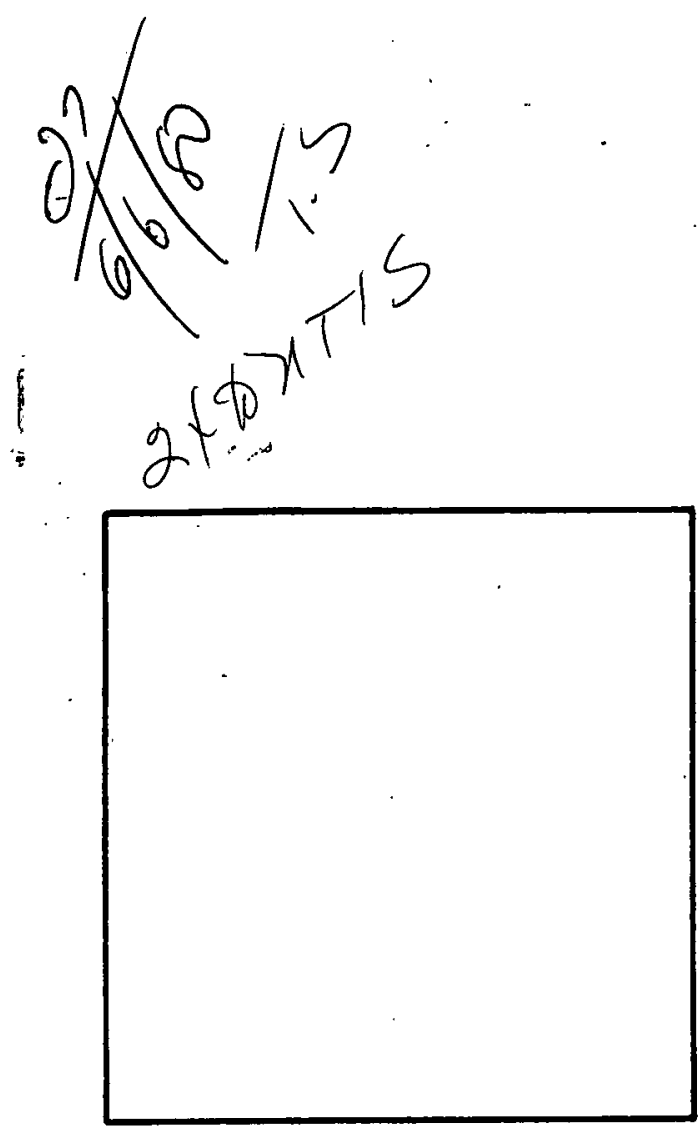

BDX-613-2412

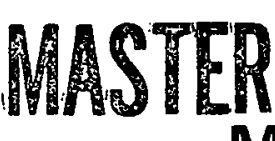

\title{
Mechanical Properties of Polystyrene Bead Encapsulation Foam
}

By D. J. Fossey

Published Mārch 1980

Topical Report

Prepared for the United States Department of Energy Under Contract Number DE-AC04-76-DP00613.

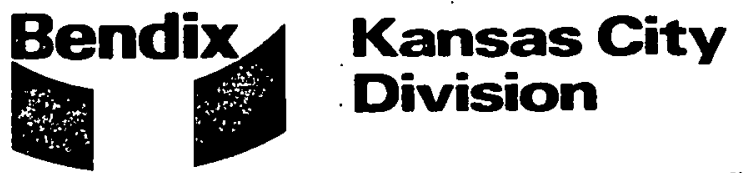




\section{DISCLAIMER}

This report was prepared as an account of work sponsored by an agency of the United States Government. Neither the United States Government nor any agency Thereof, nor any of their employees, makes any warranty, express or implied, or assumes any legal liability or responsibility for the accuracy, completeness, or usefulness of any information, apparatus, product, or process disclosed, or represents that its use would not infringe privately owned rights. Reference herein to any specific commercial product, process, or service by trade name, trademark, manufacturer, or otherwise does not necessarily constitute or imply its endorsement, recommendation, or favoring by the United States Government or any agency thereof. The views and opinions of authors expressed herein do not necessarily state or reflect those of the United States Government or any agency thereof. 


\section{DISCLAIMER}

Portions of this document may be illegible in electronic image products. Images are produced from the best available original document. 
This report was prepared as an account of work sponsored by the United States Government. Neither the United States nor the United States Department of Energy, nor any of their employees; nor any of their contractors, subcontractors, or their employees, makes any warranty, express or implied, or assumes any legal liability or responsibility for the accuracy, completeness or usefulness of any information, apparatus, product or process disclosed, or represents that its use would not infringe privately owned rights.

Printed in the United States of America

Available From the National Technical Information Service, U.S. Department of Commerce, 5285 Port Royal Road, Springfield, Virginia 22161.

Price: Microfiche $\$ 3.00$ Paper Copy $\$ \frac{\$ .50}{6.4}$ 
$\mathrm{BDX}-613-2412$

Distribution Category UC -25

MECHANICAL PROPERTIES OF

POLYSTYRENE BEAD

ENCAPSULATION FOAM

By D. J. Fossey

Published March 1980

Topical Report

D. J. Fossey, Project Leader

Project Team:

G. L. Woodburn

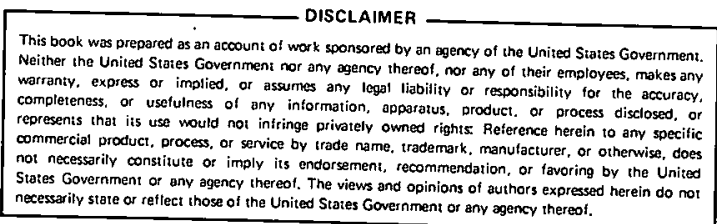

\section{Communications Services Bendix Kansas City Division}


BDX-613-2412, Topical Report, Published March 1980

Prepared by D. J. Fossey

The tensile and compressive properties of polystyrene bead foam used as an encapsulant for electronic devices were determined for densities ranging from 0.3 to $0.6 \mathrm{~g} / \mathrm{cm}^{3}$. Data were generated for these properties at $-54,25$, and $74^{\circ} \mathrm{C}$. Data previously reported for $0.2 \mathrm{~g} / \mathrm{cm}^{3}$ are included.

DDK : $\operatorname{tr} / 7$

This report was prepared as an account of work sponsored by the United States Government. Neither the United States. nor the United States Department of Energy, nor any of their employees. nor any of their contractors. subcontractors, or their employees, makes any warranty, expressed or implied or assumes any legal liability or responsibility for the accuracy. completeness or usefulness of any information. apparatus, product, or process disclosed, or represents that its use would not infringe privately owned rights.
The Bendix Corporation Kansas City Division P. O. Box 1159 Kansas City, Missouri 64141 


\section{CONTENTS}

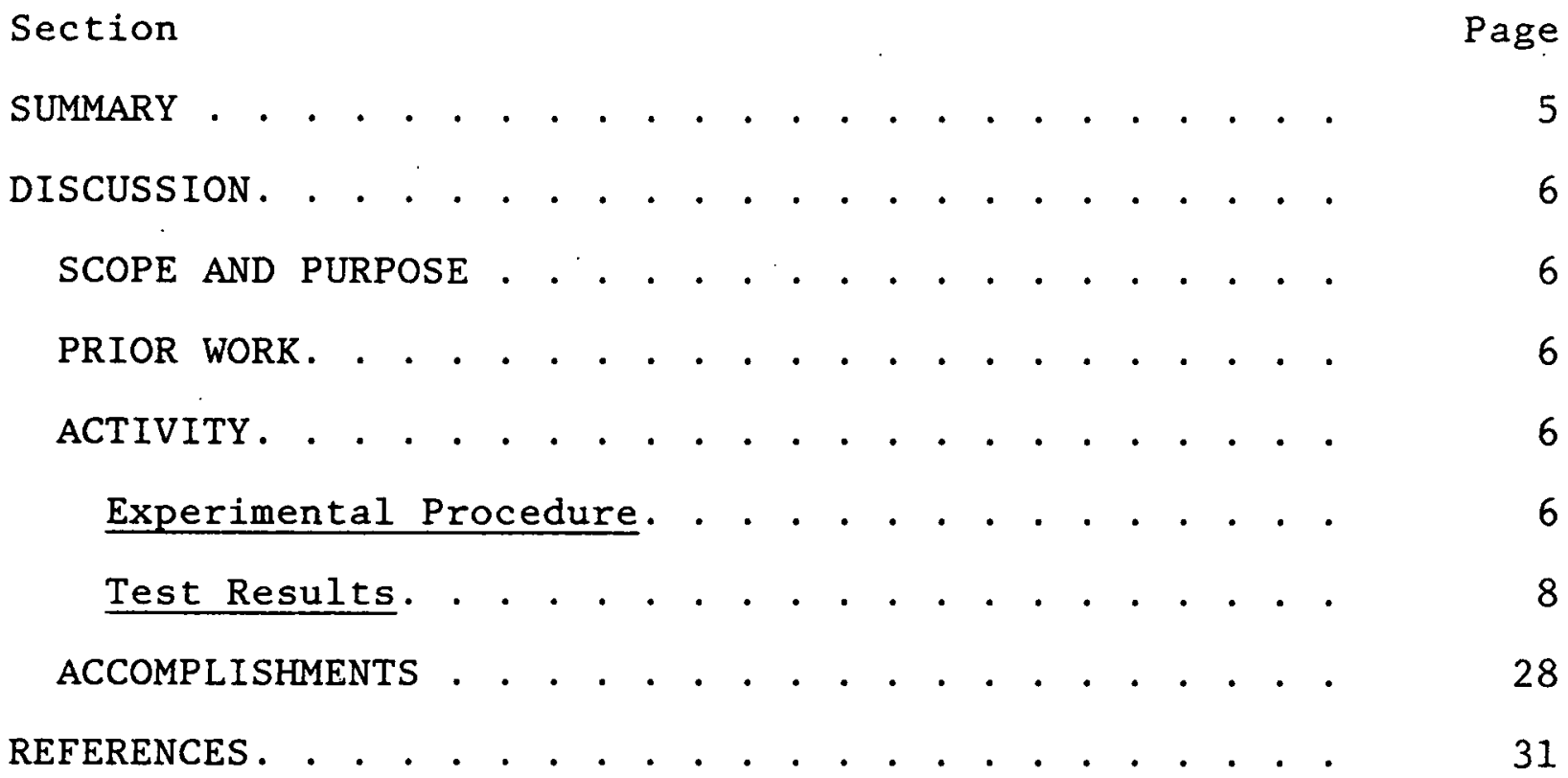




\section{ILLUSTRATIONS}

Figure

Page

1

Tensile Strength Versus Density of PSBF... .

2

Tensile Modulus Versus Density of PSBF. . .

3 Compressive Strength Versus Density of PSBF . . . . . . . . . . . . . . . . .

4 Compressive Modulus Versus Density of PSBF. .

\section{TABLES}

Number

Page

1

Process Variables. .

Test Program . . . . . . . . . . . . . .

Weight Loss Data for Heat-Aged PSBF Test

Specimens. . . . . . . . . . . . . . . . .

Test Specimen Fusion Temperature and Initial Volatile Content . . . . . . . . . . . . . .

Average Tensile Data for As-Molded PSBF. . .

Average Tensile Data for Heat-Aged PSBF. . .

Average Compressive Data for As-Molded PSBF. .

Average Compressive Data for Heat-Aged PSBF. .

Overall Average Tensile Properties of As-Molded (AM) and Heat-Aged (HA) PSBF. . .

Overall Average Compressive Properties of As-Molded (AM) and Heat-Aged (HA) PSBF ... .

Tensile Properties of $0.6 \mathrm{~g} / \mathrm{cm}^{3}$ As-Molded (AM) and Heat-Aged (HA) PSBF With Different Initial Volatile Contents. . . . . . . . . (AM) and Heat-Aged (HA) PSBF With Different Initial Volatile Contents. . . . . . . . . . 
The tensile and compressive properties of polystyrene bead foam (PSBF) were determined as a function of foam density and test temperature. The effects of the pentane content of the molded PSBF and fusion temperature on these properties were also determined. The PSBF densities investigated ranged from 0.1 to $0.6 \mathrm{~g} / \mathrm{cm}^{3}$ and were tested for their mechanical properties at -54 , 25 , and $74^{\circ} \mathrm{C}$.

Test results showed that $0.1 \mathrm{~g} / \mathrm{cm}^{3}$ PSBF cannot be processed as an encapsulant for electronic packages because of poor bead fusion. PSBF densities $0.4 \mathrm{~g} / \mathrm{cm}^{3}$ and higher continue to expand at $74^{\circ} \mathrm{C}$. Reducing the pentane or volatile content from 6 to about 3 percent in the expandable polystyrene beads before molding and increasing the fusion temperature will allow the use of higher density PSBF at $74^{\circ} \mathrm{C}$. 


\section{DISCUSSION}

\section{SCOPE AND PURPOSE}

This task was undertaken to determine the mechanical properties of polystyrene bead foam (PSBF) as a function of density ( 0.1 to $\left.0.6 \mathrm{~g} / \mathrm{cm}^{3}\right)$ and temperature $\left(-54\right.$ to $\left.74^{\circ} \mathrm{C}\right)$.

\section{PRIOR WORK}

The mechanical properties of PSBF used for structural applications have been reported for densities of 0.05 through $0.5 \mathrm{~g} / \mathrm{cm}^{3},{ }^{1,2,3}$ The only mechanical properties data that have been reported of PSBF used as an encapsulant for electronic packages were for $0.2 \mathrm{~g} / \mathrm{cm}^{3}$ density. ${ }^{4}$

\section{ACTIVITY}

\section{Experimental Procedure}

To cover the range of densities of PSBF obtainable from the expandable polystyrene (EPS) beads, the nominal densities of 0.1 , $0.3,0.4,0.5$, and $0.6 \mathrm{~g} / \mathrm{cm}^{3}$ were chosen for evaluation. A test matrix was set up to determine the tensile and compressive properties of these PSBF densities as a function of the process varibles (Table 1). Similar data had already been generated for $0.2 \mathrm{~g} / \mathrm{cm}^{3}$ PSBF. ${ }^{4}$ Three test specimens, $28.7 \mathrm{~mm}$ diameter by $25.4 \mathrm{~mm}$ high, were molded and tested for each density to determine the tensile and compressive properties at each point in this matrix. Additional test specimens were provided for volatile content determination at the time of testing. The description and process for filling the test specimen mold have been previously reported. ${ }^{4}$ The density of the PSBF was varied by preexpanding the EPS beads to a bulk density of the desired foam density. The preexpansion process involved placing a thin layer $(\sim 6 \mathrm{~mm})$ of as-received EPS beads in an oven preheated to $85^{\circ} \mathrm{C}$ and leaving them in the oven until their volume had increased to a predetermined value. The exact residence time of the EPS beads in the oven varied with the pentane content and desired bulk density. The 0.1 and $0.3 \mathrm{~g} / \mathrm{cm}^{3}$ preexpanded beads were screened ( -20 mesh, +30 mesh) before bulk density determination. The preexpanded bead with bulk densities of $0.4 \mathrm{~g} / \mathrm{cm}^{3}$ and higher were not screened. The $0.6 \mathrm{~g} / \mathrm{cm}^{3}$ PSBF was molded using as-received beads that were not preexpanded. 
Table 1. Process Variables

\begin{tabular}{ll}
\hline Variable & Value \\
\hline Fusion Temperature $\left({ }^{\circ} \mathrm{C}\right)$ & 100 and 107 \\
Conditioning & As-Molded and Heat-Aged \\
Test Temperature $\left({ }^{\circ} \mathrm{C}\right)$ & $-54,25$, and 74 \\
\hline
\end{tabular}

After filling the 12-cavity molds with the preexpanded beads, the molds were closed and placed in an oven preheated to the desired fusion temperature $\left(100\right.$ or $\left.107^{\circ} \mathrm{C}\right)$. The molded PSBF specimens were removed 20 minutes after a thermocouple placed in center of one of the cavities indicated that the beads had reached $93^{\circ} \mathrm{C}$. The length of time required for the beads to reach $93^{\circ} \mathrm{C}$ increased as the density of the preexpanded beads increased. A total of five 12-cavity moldings, or 60 test specimens, were made for each density of PSBF being evaluated. The test specimens from each density were randomly selected and numbered 1 through 48. Each specimen was weighed and measured for density determination. The specimens were then grouped for testing (Table 2). The specimens that were tested in tension were bonded between two aluminum cylinders, $51 \mathrm{~mm}$ long by $28.7 \mathrm{~mm}$ diameter, using an adhesive system cured at room temperature.

The groups of test specimens, numbers 25 through 48 , were placed in an oven to reduce the residual pentane content to a level below 0.5 weight percent. The 0.3 and $0.4 \mathrm{~g} / \mathrm{cm}^{3}$ PSBF test specimens were heat aged at $71^{\circ} \mathrm{C}$. The 0.5 and $0.6 \mathrm{~g} / \mathrm{cm}^{3}$ PSBF test specimens were initially heat aged at $55^{\circ} \mathrm{C}$ for a period of time, then transferred to an oven preheated to $71^{\circ} \mathrm{C}$. One test specimen from each density of PSBF was monitored for weight loss periodically during the heat-aging cycle. When the weight loss data indicated the residual pentane content was less than 1.5 percent for a group of test specimens, they were transferred from 55 to $71^{\circ} \mathrm{C}$ for further aging. In most cases, the heat aging was terminated when the weight loss data indicated the residual pentane content was below 0.5 percent. The residual pentane content was obtained by determining the weight loss or volatile content of a test specimen at $150^{\circ} \mathrm{C}$ for 30 minutes. All of the volatiles contained in PSBF were pentane and about 0.1 percent monomer and dimers of styrene and moisture. Therefore, pentane content and volatile content are used interchangeably in this report. The heat-aging cycles and weight loss data are given in Table 3 , and the test specimen group identification is explained in Table 4. Removal of the residual pentane in the PSBF required a much longer time 
Table 2. Test Program

\begin{tabular}{|c|c|c|}
\hline $\begin{array}{l}\text { Test } \\
\text { Specimen } \\
\text { Number }\end{array}$ & $\begin{array}{l}\text { Test } \\
\text { Temperature } \\
\left({ }^{\circ} \mathrm{C}\right)\end{array}$ & Type of Test \\
\hline \multicolumn{3}{|l|}{ As-Molded } \\
\hline 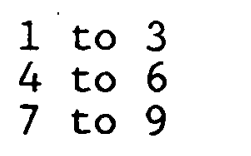 & $\begin{array}{r}-54 \\
25 \\
74\end{array}$ & $\begin{array}{l}\text { Tensile } \\
\text { Tensile } \\
\text { Tensile }\end{array}$ \\
\hline $\begin{array}{l}10 \text { to } 12 \\
13 \text { to } 15 \\
16 \text { to } 18\end{array}$ & $\begin{array}{r}-54 \\
25 \\
74\end{array}$ & $\begin{array}{l}\text { Compression } \\
\text { Compression } \\
\text { Compression }\end{array}$ \\
\hline 19 to 21 & 150 & Volatile content at time \\
\hline 22 to 24 & 150 & $\begin{array}{l}\text { Volatile content at time } \\
\text { of compression testing }\end{array}$ \\
\hline
\end{tabular}

Heat-Aged

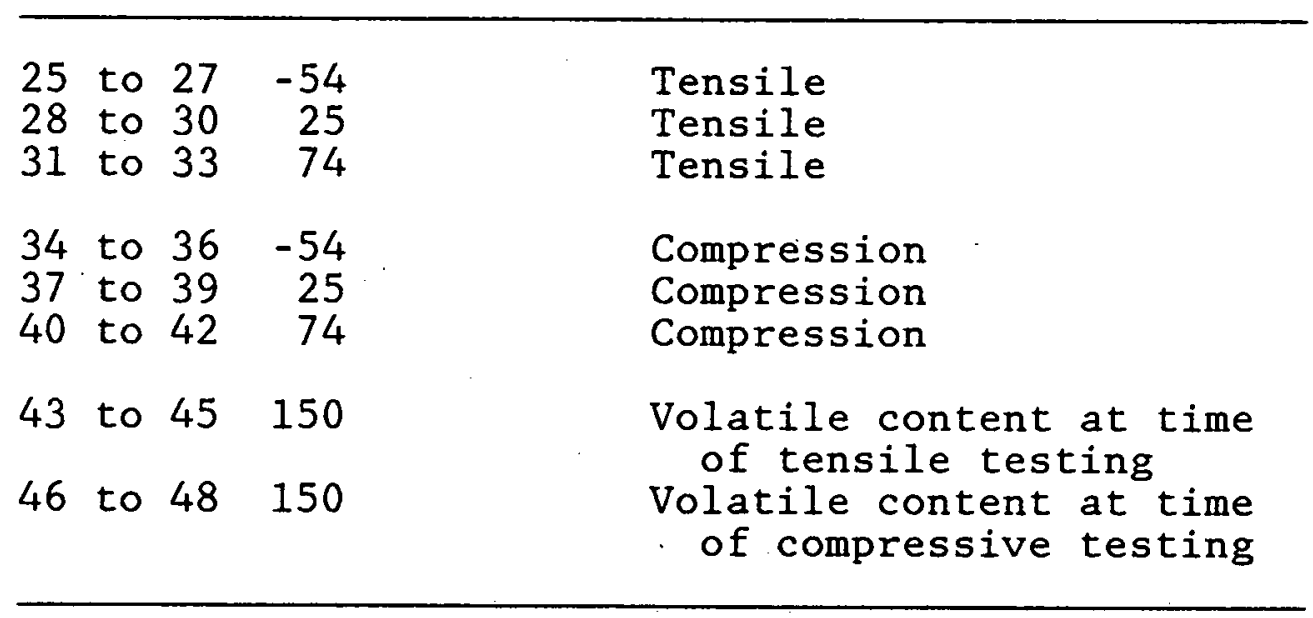

than expected, especially for the higher density PSBF. In the case of the 0.5 and $0.6 \mathrm{~g} / \mathrm{cm}^{3} \mathrm{PSBF}$, the residual pentane content was still above 0.5 percent after about 5 months of heat aging.

\section{Test Results}

The as-molded and heat-aged tensile data generated are listed in Tables 5 and 6 , respectively. Tables 7 and 8 contain the compressive data for the as-molded and heat-aged PSBF test specimens.

Text continued on page 18 . 
Table 3. Weight Loss Data for Heat-Aged PSBF Test Specimens

\begin{tabular}{|c|c|c|c|c|}
\hline \multirow{2}{*}{$\begin{array}{l}\text { Specimen } \\
\text { Group }\end{array}$} & \multicolumn{2}{|c|}{$\begin{array}{l}\text { Heat Aging Time } \\
\text { (Days) }\end{array}$} & \multirow{2}{*}{$\begin{array}{l}\text { Total } \\
\text { Weight } \\
\text { Loss } \\
\text { (Percent) }\end{array}$} & \multirow{2}{*}{$\begin{array}{l}\text { Initial } \\
\text { Volatile } \\
\text { Content } \\
\text { (Percent) }\end{array}$} \\
\hline & at $55^{\circ} \mathrm{C}$ & at $71^{\circ} \mathrm{C}$ & & \\
\hline $\begin{array}{l}3 \mathrm{~A} \\
3 \mathrm{~B} \\
3 \mathrm{~A}\end{array}$ & $\begin{array}{r}0 \\
0 \\
14\end{array}$ & $\begin{array}{r}109 \\
109 \\
48\end{array}$ & $\begin{array}{l}2.42 \\
2.17 \\
2.18\end{array}$ & $\begin{array}{l}2.54 \\
2.32 \\
2.40\end{array}$ \\
\hline $\begin{array}{l}4 A \\
4 B\end{array}$ & $\begin{array}{l}0 \\
0\end{array}$ & $\begin{array}{l}108 \\
108\end{array}$ & $\begin{array}{l}2.88 \\
2.89\end{array}$ & $\begin{array}{l}3.10 \\
3.10\end{array}$ \\
\hline $\begin{array}{l}5 \mathrm{~A} \\
5 \mathrm{~B}\end{array}$ & $\begin{array}{l}56 \\
56\end{array}$ & $\begin{array}{l}89 \\
89\end{array}$ & $\begin{array}{l}2.73 \\
2.45\end{array}$ & $\begin{array}{l}3.02 \\
3.02\end{array}$ \\
\hline $\begin{array}{l}6 \mathrm{~A} \\
6 \mathrm{~B} \\
6 \mathrm{D} \\
6 \mathrm{E} \\
6 \mathrm{~F}\end{array}$ & $\begin{array}{r}167 \\
167 \\
20 \\
19 \\
19\end{array}$ & $\begin{array}{r}2 \\
2 \\
74 \\
107 \\
74\end{array}$ & $\begin{array}{l}1.94 \\
2.65 \\
2.31 \\
2.41 \\
2.21\end{array}$ & $\begin{array}{l}3.85 \\
3.89 \\
2.46 \\
2.77 \\
2.31\end{array}$ \\
\hline
\end{tabular}

*See Table 4 for definition of test specimen identification. **Determined by testing an extra test specimen for volatile content.

Table 4. Test Specimen Fusion Temperature and Initial Volatile Content

\begin{tabular}{llll}
\hline $\begin{array}{l}\text { Specimen } \\
\text { Group }\end{array}$ & $\begin{array}{l}\text { Nominal } \\
\text { Density } \\
\left(\mathrm{g} / \mathrm{cm}^{3}\right)\end{array}$ & $\begin{array}{l}\text { Initial Volatile } \\
\text { Content of Preexpanded } \\
\text { Beads (Percent) }\end{array}$ & $\begin{array}{l}\text { Fusion } \\
\text { Temperature } \\
\left({ }^{\circ} \mathrm{C}\right)\end{array}$ \\
\hline 3A & 0.3 & 4.35 & 100 \\
3B & 0.3 & 4.35 & 107 \\
3A & 0.3 & 4.20 & 100 \\
4A & 0.4 & 4.49 & 100 \\
4B & 0.4 & 4.49 & 107 \\
5A & 0.5 & 4.66 & 100 \\
5B & 0.5 & 4.66 & 107 \\
6A & 0.6 & 6.04 & 100 \\
6B & 0.6 & 6.04 & 107 \\
6C & 0.6 & 2.70 & 100 \\
6D & 0.6 & 2.70 & 107 \\
6E & 0.6 & 3.18 & 100 \\
6F & 0.6 & 3.18 & 107 \\
\hline
\end{tabular}


Table 5. Average Tensile Data for As-Molded PSBF

\begin{tabular}{|c|c|c|c|c|c|c|}
\hline $\begin{array}{l}\text { Test } \\
\text { Group }\end{array}$ & $\begin{array}{l}\text { Density } \\
\left(\mathrm{g} / \mathrm{cm}^{3}\right)\end{array}$ & $\begin{array}{l}\text { Volatile } \\
\text { Content } \\
\text { (Percent) }\end{array}$ & $\begin{array}{l}\text { Test } \\
\text { Temperature } \\
\left({ }^{\circ} \mathrm{C}\right)\end{array}$ & $\begin{array}{l}\text { Ultimate } \\
\text { Strength } \\
\text { (MPa) }\end{array}$ & $\begin{array}{l}\text { Modulus } \\
(\mathrm{MPa})\end{array}$ & $\begin{array}{l}\text { Elongation } \\
\text { (Percent) }\end{array}$ \\
\hline $\begin{array}{l}3 A \\
3 B \\
3 A \\
3 A \\
3 B\end{array}$ & $\begin{array}{l}0.301 \\
0.299 \\
0.299 \\
0.299 \\
0.298\end{array}$ & $\begin{array}{l}1.35 \\
1.11 \\
1.93 \\
1.44 \\
1.02\end{array}$ & $\begin{array}{r}-54 \\
-54 \\
-54 \\
25 \\
25\end{array}$ & $\begin{array}{l}0.30 \\
0.35 \\
0.14 \\
0.39 \\
0.57\end{array}$ & $\begin{array}{l}163 \\
306 \\
165 \\
337 \\
306\end{array}$ & $\begin{array}{l}0.19 \\
0.13 \\
0.16 \\
0.16 \\
0.18\end{array}$ \\
\hline $\begin{array}{l}3 A^{-} \\
3 A \\
3 B \\
3 A^{-}\end{array}$ & $\begin{array}{l}0.298 \\
0.285 \\
0.304 \\
0.304\end{array}$ & $\begin{array}{l}1.92 \\
1.51 \\
1.06 \\
1.84\end{array}$ & $\begin{array}{l}25 \\
74 \\
74 \\
74\end{array}$ & $\begin{array}{l}0.32 \\
0.44 \\
0.73 \\
0.37\end{array}$ & $\begin{array}{l}269 \\
164 \\
334 \\
221\end{array}$ & $\begin{array}{l}0.14 \\
0.25 \\
0.32 \\
0.25\end{array}$ \\
\hline $\begin{array}{l}4 \mathrm{~A} \\
4 \mathrm{~B} \\
4 \mathrm{~A} \\
4 \mathrm{~B} \\
4 \mathrm{~A} \\
4 \mathrm{~B}\end{array}$ & $\begin{array}{l}0.386 \\
0.393 \\
0.392 \\
0.393 \\
0.386 \\
0.389\end{array}$ & $\begin{array}{l}2.32 \\
2.41 \\
2.36 \\
2.20 \\
2.53 \\
2.36\end{array}$ & $\begin{array}{r}-54 \\
-54 \\
25 \\
25 \\
74 \\
74\end{array}$ & $\begin{array}{l}0.78 \\
1.99 \\
1.92 \\
2.69 \\
1.47 \\
1.81\end{array}$ & $\begin{array}{l}509 \\
623 \\
583 \\
533 \\
275 \\
259\end{array}$ & $\begin{array}{l}0.40 \\
0.38 \\
0.34 \\
0.52 \\
0.74 \\
1.44\end{array}$ \\
\hline $\begin{array}{l}5 \mathrm{~A} \\
5 \mathrm{~B} \\
5 \mathrm{~A} \\
5 \mathrm{~B} \\
5 \mathrm{~A} \\
5 \mathrm{~B}\end{array}$ & $\begin{array}{l}0.493 \\
0.496 \\
0.490 \\
0.491 \\
0.493 \\
0.489\end{array}$ & $\begin{array}{l}2.79 \\
2.72 \\
2.72 \\
2.73 \\
2.60 \\
2.64\end{array}$ & $\begin{array}{r}-54 \\
-54 \\
25 \\
25 \\
74 \\
74\end{array}$ & $\begin{array}{l}1.64 \\
2.88 \\
3.49 \\
4.34 \\
1.30 \\
0.91\end{array}$ & $\begin{array}{l}862 \\
793 \\
750 \\
774 \\
190 \\
129\end{array}$ & $\begin{array}{l}0.20 \\
0.37 \\
0.49 \\
0.56 \\
1.84 \\
2.08\end{array}$ \\
\hline $\begin{array}{l}6 A \\
6 B \\
6 A \\
6 B \\
6 A\end{array}$ & $\begin{array}{l}0.600 \\
0.586 \\
0.597 \\
0.584 \\
0.601\end{array}$ & $\begin{array}{l}3.10 \\
3.82 \\
3.94 \\
3.97 \\
3.10\end{array}$ & $\begin{array}{r}-54 \\
-54 \\
25 \\
25 \\
74\end{array}$ & $\begin{array}{l}1.86 * \\
1.41 * \\
5.41 \\
5.17 \\
0.03\end{array}$ & $\begin{array}{r}1090 \\
1160 \\
669 \\
669 \\
34\end{array}$ & $\begin{array}{l}0.15 \\
0.18 \\
1.11 \\
1.12 \\
1.63\end{array}$ \\
\hline
\end{tabular}


Table 5 Continued. Average Tensile Data for As-Molded PSBF

\begin{tabular}{|c|c|c|c|c|c|c|}
\hline $\begin{array}{l}\text { Test } \\
\text { Group }\end{array}$ & $\begin{array}{l}\text { Density } \\
\left(\mathrm{g} / \mathrm{cm}^{3}\right)\end{array}$ & $\begin{array}{l}\text { Volatile } \\
\text { Content } \\
\text { (Percent) }\end{array}$ & $\begin{array}{l}\text { Test } \\
\text { Temperature } \\
\left({ }^{\circ} \mathrm{C}\right)\end{array}$ & $\begin{array}{l}\text { Ultimate } \\
\text { Strength } \\
\text { (MPa) }\end{array}$ & $\begin{array}{l}\text { Modulus } \\
\text { (MPa) }\end{array}$ & $\begin{array}{l}\text { Elongation } \\
\text { (Percent) }\end{array}$ \\
\hline $\begin{array}{l}6 \mathrm{~B} \\
6 \mathrm{C} \\
6 \mathrm{D} \\
6 \mathrm{C} \\
6 \mathrm{D}\end{array}$ & $\begin{array}{l}0.580 \\
0.618 \\
0.616 \\
0.621 \\
0.621\end{array}$ & $\begin{array}{l}3.63 \\
2.25 \\
2.19 \\
2.28 \\
2.25\end{array}$ & $\begin{array}{r}74 \\
-54 \\
-54 \\
25 \\
25\end{array}$ & $\begin{array}{l}0.05 \\
x+1 \\
t+1 \\
0.21 \\
0.85\end{array}$ & $\begin{array}{c}83 \\
-- \\
-- \\
646 \\
944\end{array}$ & $\begin{array}{c}1.02 \\
-- \\
-- \\
0.03 \\
0.27\end{array}$ \\
\hline $\begin{array}{l}6 C \\
6 D \\
6 E \\
6 F \\
6 E .\end{array}$ & $\begin{array}{l}0.620 \\
0.620 \\
0.614 \\
0.608 \\
0.613\end{array}$ & $\begin{array}{l}2.26 \\
2.22 \\
2.53 \\
2.08 \\
2.47\end{array}$ & $\begin{array}{r}74 \\
74 \\
-54 \\
-54 \\
25\end{array}$ & $\begin{array}{l}\frac{1}{\pi} \\
0.18 \\
+x \\
0.77 \\
1.48\end{array}$ & $\begin{array}{c}-- \\
575 \\
-- \\
1080 \\
1129\end{array}$ & $\begin{array}{l}-\overline{-} \\
0.11 \\
-\overline{11} \\
0.11 \\
0.16\end{array}$ \\
\hline $\begin{array}{l}6 \mathrm{~F} \\
6 \mathrm{E} \\
6 \mathrm{~F}\end{array}$ & $\begin{array}{l}0.606 \\
0.618 \\
0.607\end{array}$ & $\begin{array}{l}2.14 \\
2.48 \\
2.05\end{array}$ & $\begin{array}{l}25 \\
74 \\
74\end{array}$ & $\begin{array}{l}3.00 \\
0.17 \\
2.30\end{array}$ & $\begin{array}{r}1080 \\
190 \\
621\end{array}$ & $\begin{array}{l}0.28 \\
0.15 \\
0.50\end{array}$ \\
\hline
\end{tabular}

¿Failure was between adhesive and aluminum plug. $\star \star$ Specimen broke during handling 
Table 6. Average Tensile Data for Heat-Aged PSBF

\begin{tabular}{|c|c|c|c|c|c|c|}
\hline $\begin{array}{l}\text { Test } \\
\text { Group }\end{array}$ & $\begin{array}{l}\text { Density } \\
\left(\mathrm{g} / \mathrm{cm}^{3}\right)\end{array}$ & $\begin{array}{l}\text { Volatile } \\
\text { Content } \\
\text { (Percent) }\end{array}$ & $\begin{array}{l}\text { Test } \\
\text { Temperature } \\
\left({ }^{\circ} \mathrm{C}\right)\end{array}$ & $\begin{array}{l}\text { Ultimate } \\
\text { Strength } \\
\text { (MPa) }\end{array}$ & $\begin{array}{l}\text { Modulus } \\
\text { (MPa) }\end{array}$ & $\begin{array}{l}\text { Elongation } \\
\text { (Percent) }\end{array}$ \\
\hline $\begin{array}{l}3 \mathrm{~A} \\
3 \mathrm{~B} \\
3 \mathrm{~A} \\
3 \mathrm{~A} \\
3 \mathrm{~B}\end{array}$ & $\begin{array}{l}0.293 \\
0.297 \\
0.291 \\
0.296 \\
0.301\end{array}$ & $\begin{array}{l}0.18 \\
0.13 \\
0.20 \\
0.16 \\
0.12\end{array}$ & $\begin{array}{r}-54 \\
-54 \\
-54 \\
25 \\
25\end{array}$ & $\begin{array}{l}0.32 \\
0.50 \\
0.23 \\
0.64 \\
0.89\end{array}$ & $\begin{array}{c}-- \\
340.6 \\
362.7 \\
318.5 \\
340.6\end{array}$ & $\begin{array}{l}-- \\
0.14 \\
0.04 \\
0.20 \\
0.27\end{array}$ \\
\hline $\begin{array}{l}3 A^{-} \\
3 A \\
3 B \\
3 A^{-}\end{array}$ & $\begin{array}{l}0.293 \\
0.294 \\
0.301 \\
0.299\end{array}$ & $\begin{array}{l}0.25 \\
0.18 \\
0.13 \\
0.24\end{array}$ & $\begin{array}{l}25 \\
74 \\
74 \\
74\end{array}$ & $\begin{array}{l}0.61 \\
0.58 \\
0.90 \\
0.56\end{array}$ & $\begin{array}{l}324.7 \\
309.6 \\
322.0 \\
288.2\end{array}$ & $\begin{array}{l}0.19 \\
0.18 \\
0.29 \\
0.18\end{array}$ \\
\hline $\begin{array}{l}4 \mathrm{~A} \\
4 \mathrm{~B} \\
4 \mathrm{~A} \\
4 \mathrm{~B} \\
4 \mathrm{~A} \\
4 \mathrm{~B}\end{array}$ & $\begin{array}{l}0.375 \\
0.389 \\
0.383 \\
0.385 \\
0.381 \\
0.377\end{array}$ & $\begin{array}{l}0.23 \\
0.23 \\
0.22 \\
0.23 \\
0.23 \\
0.25\end{array}$ & $\begin{array}{r}-54 \\
-54 \\
25 \\
25 \\
74 \\
74\end{array}$ & $\begin{array}{l}1.62 \\
1.72 \\
2.26 \\
3.19 \\
2.33 \\
2.54\end{array}$ & $\begin{array}{l}647.4 \\
724.0 \\
524.7 \\
565.4 \\
464.0 \\
521.2\end{array}$ & $\begin{array}{l}0.24 \\
0.26 \\
0.46 \\
0.59 \\
0.53 \\
0.51\end{array}$ \\
\hline $\begin{array}{l}5 \mathrm{~A} \\
5 \mathrm{~B} \\
5 \mathrm{~A} \\
5 \mathrm{~B} \\
5 \mathrm{~A} \\
5 \mathrm{~B}\end{array}$ & $\begin{array}{l}0.484 \\
0.487 \\
0.485 \\
0.490 \\
0.491 \\
0.493\end{array}$ & $\begin{array}{l}0.27 \\
0.71 \\
0.27 \\
0.67 \\
0.42 \\
0.61\end{array}$ & $\begin{array}{r}-54 \\
-54 \\
25 \\
25 \\
74 \\
74\end{array}$ & $\begin{array}{l}2.05 \\
2.41 \\
3.41 \\
4.18 \\
3.24 \\
3.17\end{array}$ & $\begin{array}{r}855.0 \\
841.1 \\
1407.0 \\
1192.8 \\
1323.8 \\
1454.8\end{array}$ & $\begin{array}{l}0.24 \\
0.28 \\
0.43 \\
0.61 \\
0.53 \\
0.51\end{array}$ \\
\hline $\begin{array}{l}6 \mathrm{~A} \\
6 \mathrm{~B} \\
6 \mathrm{D} \\
6 \mathrm{E} \\
6 \mathrm{~F} \\
6 \mathrm{D}\end{array}$ & $\begin{array}{l}0.614 \\
0.608 \\
0.619 \\
0.622 \\
0.607 \\
0.623\end{array}$ & $\begin{array}{l}1.57 \\
1.83 \\
0.16 \\
0.15 \\
0.16 \\
0.15\end{array}$ & $\begin{array}{r}25 \\
25 \\
-54 \\
-54 \\
-54 \\
25\end{array}$ & $\begin{array}{l}7.05 \\
6.81 \\
0.10 \\
0.36 \\
0.98 \\
1.38\end{array}$ & $\begin{array}{r}875.6 \\
910.1 \\
882.5 \\
1254.9 \\
1213.5 \\
1151.4\end{array}$ & $\begin{array}{l}0.94 \\
0.87 \\
0.07 \\
0.03 \\
0.11 \\
0.13\end{array}$ \\
\hline
\end{tabular}


Table 6 Continued. Average Tensile Data for Heat-Aged PSBF

\begin{tabular}{lllllll}
\hline $\begin{array}{l}\text { Test } \\
\text { Group }\end{array}$ & $\begin{array}{l}\text { Density } \\
\left(\mathrm{g} / \mathrm{cm}^{3}\right)\end{array}$ & $\begin{array}{l}\text { Volatile } \\
\text { Content } \\
(\text { Percent })\end{array}$ & $\begin{array}{l}\text { Test } \\
\text { Temperature } \\
\left({ }^{\circ} \mathrm{C}\right)\end{array}$ & $\begin{array}{l}\text { Ultimate } \\
\text { Strength } \\
\text { (MPa) }\end{array}$ & $\begin{array}{l}\text { Modulus } \\
(\mathrm{MPa})\end{array}$ & $\begin{array}{l}\text { Elongation } \\
\text { (Percent) }\end{array}$ \\
\hline 6E & 0.625 & 0.13 & 25 & 1.21 & 1034.2 & 0.11 \\
$6 \mathrm{~F}$ & 0.610 & 0.14 & 25 & 2.34 & 1261.7 & 0.19 \\
$6 \mathrm{D}$ & 0.621 & 0.16 & 74 & 0.81 & 889.4 & 0.11 \\
$6 \mathrm{E}$ & 0.621 & 0.13 & 74 & 0.95 & 724.0 & 0.14 \\
$6 \mathrm{~F}$ & 0.614 & 0.18 & 74 & 1.79 & 889.4 & 0.27 \\
\hline
\end{tabular}


Table 7. Average Compressive Data for As-Molded PSBF

\begin{tabular}{|c|c|c|c|c|c|c|}
\hline $\begin{array}{l}\text { Test } \\
\text { Group }\end{array}$ & $\begin{array}{l}\text { Density } \\
\left(\mathrm{g} / \mathrm{cm}^{3}\right)\end{array}$ & $\begin{array}{l}\text { Volatile } \\
\text { Content } \\
\text { (Percent) }\end{array}$ & $\begin{array}{l}\text { Test } \\
\text { Temperature } \\
\left({ }^{\circ} \mathrm{C}\right)\end{array}$ & $\begin{array}{l}\text { Ultimate } \\
\text { Strength } \\
\text { (MPa) }\end{array}$ & $\begin{array}{l}\text { Modulus } \\
(\mathrm{MPa})\end{array}$ & $\begin{array}{l}\text { Deflection } \\
\text { (Percent) }\end{array}$ \\
\hline $\begin{array}{l}3 \mathrm{~A} \\
3 \mathrm{~B} \\
3 \mathrm{~A} \\
3 \mathrm{~A} \\
3 \mathrm{~B}\end{array}$ & $\begin{array}{l}0.296 \\
0.296 \\
0.293 \\
0.295 \\
0.299\end{array}$ & $\begin{array}{l}1.50 \\
1.17 \\
1.93 \\
2.03 \\
1.38\end{array}$ & $\begin{array}{r}-54 \\
-54 \\
-54 \\
25 \\
25\end{array}$ & $\begin{array}{l}5.54 \\
6.12 \\
6.01 \\
3.88 \\
4.22\end{array}$ & $\begin{array}{l}317 \\
317 \\
344 \\
248 \\
283\end{array}$ & $\begin{array}{l}2.1 \\
2.7 \\
2.5 \\
2.7 \\
3.1\end{array}$ \\
\hline $\begin{array}{l}3 A^{\circ} \\
3 A \\
3 B \\
3 A^{\circ} \\
4 A^{\circ}\end{array}$ & $\begin{array}{l}0.297 \\
0.298 \\
0.295 \\
0.290 \\
0.381\end{array}$ & $\begin{array}{l}1.92 \\
1.89 \\
1.52 \\
1.84 \\
2.44\end{array}$ & $\begin{array}{r}25 \\
74 \\
74 \\
74 \\
-54\end{array}$ & $\begin{array}{r}4.16 \\
2.36 \\
2.60 \\
2.32 \\
12.55\end{array}$ & $\begin{array}{l}310 \\
179 \\
193 \\
179 \\
441\end{array}$ & $\begin{array}{l}2.7 \\
2.5 \\
2.4 \\
2.4 \\
3.9\end{array}$ \\
\hline $\begin{array}{l}4 B \\
4 A \\
4 B \\
4 A \\
4 B\end{array}$ & $\begin{array}{l}0.378 \\
0.379 \\
0.387 \\
0.395 \\
0.392\end{array}$ & $\begin{array}{l}2.43 \\
2.98 \\
3.00 \\
2.86 \\
2.97\end{array}$ & $\begin{array}{r}-54 \\
25 \\
25 \\
74 \\
74\end{array}$ & $\begin{array}{r}13.58 \\
8.14 \\
8.00 \\
2.12 \\
2.03\end{array}$ & $\begin{array}{r}455 \\
421 \\
414 \\
120 \\
90\end{array}$ & $\begin{array}{l}4.7 \\
3.6 \\
3.7 \\
2.7 \\
3.0\end{array}$ \\
\hline $\begin{array}{l}5 \mathrm{~A} \\
5 \mathrm{~B} \\
5 \mathrm{~A} \\
5 \mathrm{~B} \\
5 \mathrm{~A}\end{array}$ & $\begin{array}{l}0.497 \\
0.487 \\
0.500 \\
0.495 \\
0.500\end{array}$ & $\begin{array}{l}2.74 \\
2.79 \\
2.80 \\
2.76 \\
2.81\end{array}$ & $\begin{array}{r}-54 \\
-54 \\
25 \\
25 \\
74\end{array}$ & $\begin{array}{r}20.20 \\
22.13 \\
13.03 \\
12.89 \\
0.88\end{array}$ & $\begin{array}{r}538 \\
524 \\
655 \\
669 \\
14\end{array}$ & $\begin{array}{l}4.4 \\
5.5 \\
2.8 \\
3.1 \\
--\end{array}$ \\
\hline $\begin{array}{l}5 B \\
6 A \\
6 B \\
6 A \\
6 B\end{array}$ & $\begin{array}{l}0.497 \\
0.595 \\
0.586 \\
0.601 \\
0.587\end{array}$ & $\begin{array}{l}2.81 \\
3.75 \\
4.11 \\
3.58 \\
3.57\end{array}$ & $\begin{array}{r}74 \\
-54 \\
-54 \\
25 \\
25\end{array}$ & $\begin{array}{r}0.58 \\
29.92 \\
28.61 \\
15.65 \\
14.54\end{array}$ & $\begin{array}{r}7 \\
1034 \\
931 \\
717 \\
648\end{array}$ & $\begin{array}{l}-- \\
4.2 \\
5.2 \\
3.9 \\
3.8\end{array}$ \\
\hline
\end{tabular}


Table 7 Continued. Average Compressive Data for As-Molded PSBF

\begin{tabular}{|c|c|c|c|c|c|c|}
\hline $\begin{array}{l}\text { Test } \\
\text { Group }\end{array}$ & $\begin{array}{l}\text { Density } \\
\left(\mathrm{g} / \mathrm{cm}^{3}\right)\end{array}$ & $\begin{array}{l}\text { Volatile } \\
\text { Content } \\
\text { (Percent) }\end{array}$ & $\begin{array}{l}\text { Test } \\
\text { Temperature } \\
\left({ }^{\circ} \mathrm{C}\right)\end{array}$ & $\begin{array}{l}\text { Ultimate } \\
\text { Strength } \\
\text { (MPa) }\end{array}$ & $\begin{array}{l}\text { Modulus } \\
(\mathrm{MPa})\end{array}$ & $\begin{array}{l}\text { Deflection } \\
\text { (Percent) }\end{array}$ \\
\hline $\begin{array}{l}6 A \\
6 B \\
6 C \\
6 D \\
6 C\end{array}$ & $\begin{array}{l}0.602 \\
0.589 \\
0.618 \\
0.616 \\
0.618\end{array}$ & $\begin{array}{l}3.77 \\
4.06 \\
2.31 \\
2.22 \\
2.30\end{array}$ & $\begin{array}{r}74 \\
74 \\
-54 \\
-54 \\
25\end{array}$ & $\begin{array}{l}0.03 \\
0.02 \\
0.50 \\
4.77 \\
1.69\end{array}$ & $\begin{array}{l}* \\
\star \\
2 \\
28 \\
400 \\
607\end{array}$ & $\begin{array}{l}-- \\
-- \\
1.3 \\
2.1 \\
0.2\end{array}$ \\
\hline $\begin{array}{l}6 \mathrm{D} \\
6 \mathrm{C} \\
6 \mathrm{D} \\
6 \mathrm{E} \\
6 \mathrm{~F}\end{array}$ & $\begin{array}{l}0.618 \\
0.617 \\
0.617 \\
0.618 \\
0.612\end{array}$ & $\begin{array}{l}2.22 \\
2.32 \\
2.23 \\
2.51 \\
2.21\end{array}$ & $\begin{array}{r}25 \\
74 \\
74 \\
-54 \\
-54\end{array}$ & $\begin{array}{r}6.09 \\
0.68 \\
3.08 \\
4.77 \\
18.47\end{array}$ & $\begin{array}{l}738 \\
138 \\
407 \\
510 \\
745\end{array}$ & $\begin{array}{l}0.9 \\
0.9 \\
2.1 \\
2.1 \\
2.2\end{array}$ \\
\hline $\begin{array}{l}6 \mathrm{E} \\
6 \mathrm{~F} \\
6 \mathrm{E} \\
6 \mathrm{~F}\end{array}$ & $\begin{array}{l}0.615 \\
0.611 \\
0.617 \\
0.610\end{array}$ & $\begin{array}{l}2.59 \\
2.22 \\
2.53 \\
2.16\end{array}$ & $\begin{array}{l}25 \\
25 \\
74 \\
74\end{array}$ & $\begin{array}{r}6.69 \\
15.38 \\
3.57 \\
4.96\end{array}$ & $\begin{array}{l}786 \\
986 \\
186 \\
200\end{array}$ & $\begin{array}{l}0.9 \\
1.6 \\
3.4 \\
3.0\end{array}$ \\
\hline
\end{tabular}

*Modulus too low to determine. 
Table 8. Average Compressive Data for Heat-Aged PSBF

\begin{tabular}{|c|c|c|c|c|c|c|}
\hline $\begin{array}{l}\text { Test } \\
\text { Group }\end{array}$ & $\begin{array}{l}\text { Density } \\
\left(\mathrm{g} / \mathrm{cm}^{3}\right)\end{array}$ & $\begin{array}{l}\text { Volatile } \\
\text { Content } \\
\text { (Percent) }\end{array}$ & $\begin{array}{l}\text { Test } \\
\text { Temperature } \\
\left({ }^{\circ} \mathrm{C}\right)\end{array}$ & $\begin{array}{l}\text { Ultimate } \\
\text { Strength } \\
(\mathrm{MPa})\end{array}$ & $\begin{array}{l}\text { Modulus } \\
\text { (MPa) }\end{array}$ & $\begin{array}{l}\text { Deflection } \\
\text { (Percent) }\end{array}$ \\
\hline $\begin{array}{l}3 \mathrm{~A} \\
3 \mathrm{~B} \\
3 \mathrm{~A} \\
3 \mathrm{~A} \\
3 \mathrm{~B}\end{array}$ & $\begin{array}{l}0.303 \\
0.292 \\
0.294 \\
0.307 \\
0.299\end{array}$ & $\begin{array}{l}0.17 \\
0.13 \\
0.25 \\
0.20 \\
0.16\end{array}$ & $\begin{array}{r}-54 \\
-54 \\
-54 \\
25 \\
25\end{array}$ & $\begin{array}{l}5.72 \\
5.78 \\
5.77 \\
4.61 \\
5.01\end{array}$ & $\begin{array}{l}287.5 \\
273.0 \\
279.9 \\
315.1 \\
315.1\end{array}$ & $\begin{array}{l}2.7 \\
3.0 \\
2.7 \\
2.6 \\
3.3\end{array}$ \\
\hline $\begin{array}{l}3 A^{-} \\
3 A \\
3 B \\
3 A^{-}\end{array}$ & $\begin{array}{l}0.293 \\
0.295 \\
0.301 \\
0.296\end{array}$ & $\begin{array}{l}0.24 \\
0.19 \\
0.15 \\
0.22\end{array}$ & $\begin{array}{l}25 \\
74 \\
74 \\
74\end{array}$ & $\begin{array}{l}4.59 \\
3.10 \\
3.54 \\
3.39\end{array}$ & $\begin{array}{l}279.9 \\
297.2 \\
266.1 \\
235.1\end{array}$ & $\begin{array}{l}2.7 \\
2.1 \\
2.6 \\
2.6\end{array}$ \\
\hline $\begin{array}{l}4 \mathrm{~A} \\
4 \mathrm{~B} \\
4 \mathrm{~A} \\
4 \mathrm{~B} \\
4 \mathrm{~A} \\
4 \mathrm{~B}\end{array}$ & $\begin{array}{l}0.377 \\
0.386 \\
0.378 \\
0.393 \\
0.384 \\
0.392\end{array}$ & $\begin{array}{l}0.20 \\
0.20 \\
0.22 \\
0.22 \\
0.23 \\
0.24\end{array}$ & $\begin{array}{r}-54 \\
-54 \\
25 \\
25 \\
74 \\
74\end{array}$ & $\begin{array}{r}13.03 \\
14.96 \\
10.34 \\
11.58 \\
7.44 \\
7.22\end{array}$ & $\begin{array}{l}494.4 \\
508.8 \\
504.7 \\
499.2 \\
448.2 \\
399.2\end{array}$ & $\begin{array}{l}3.3 \\
4.2 \\
3.3 \\
3.8 \\
3.1 \\
3.2\end{array}$ \\
\hline $\begin{array}{l}5 \mathrm{~A} \\
5 \mathrm{~B} \\
5 \mathrm{~A} \\
5 \mathrm{~B} \\
5 \mathrm{~A} \\
5 \mathrm{~B}\end{array}$ & $\begin{array}{l}0.497 \\
0.492 \\
0.493 \\
0.492 \\
0.493 \\
0.493\end{array}$ & $\begin{array}{l}0.29 \\
0.89 \\
0.34 \\
0.83 \\
0.27 \\
0.57\end{array}$ & $\begin{array}{r}-54 \\
-54 \\
25 \\
25 \\
74 \\
74\end{array}$ & $\begin{array}{r}20.62 \\
22.96 \\
17.03 \\
15.31 \\
10.82 \\
9.45\end{array}$ & $\begin{array}{l}979.1 \\
758.4 \\
737.7 \\
675.7 \\
613.6 \\
441.3\end{array}$ & $\begin{array}{l}3.1 \\
3.7 \\
3.0 \\
4.0 \\
2.6 \\
2.9\end{array}$ \\
\hline $\begin{array}{l}6 \mathrm{~A} \\
6 \mathrm{~B} \\
6 \mathrm{D} \\
6 \mathrm{E} \\
6 \mathrm{~F} \\
6 \mathrm{D}\end{array}$ & $\begin{array}{l}0.612 \\
0.610 \\
0.622 \\
0.614 \\
0.612 \\
0.617\end{array}$ & $\begin{array}{l}1.58 \\
1.80 \\
0.20 \\
0.19 \\
0.20 \\
0.21\end{array}$ & $\begin{array}{r}25 \\
25 \\
-54 \\
-54 \\
-54 \\
25\end{array}$ & $\begin{array}{r}20.68 \\
18.72 \\
11.31 \\
14.31 \\
19.99 \\
5.52\end{array}$ & $\begin{array}{r}923.3 \\
764.8 \\
827.4 \\
1034.2 \\
1151.4 \\
765.3\end{array}$ & $\begin{array}{l}4.4 \\
4.9 \\
1.4 \\
1.4 \\
2.0 \\
0.8\end{array}$ \\
\hline
\end{tabular}


Table 8 Continued. Average Compressive Data for Heat-Aged PSBF

\begin{tabular}{lllllll}
\hline $\begin{array}{l}\text { Test } \\
\text { Group }\end{array}$ & $\begin{array}{l}\text { Density } \\
\left(\mathrm{g} / \mathrm{cm}^{3}\right)\end{array}$ & $\begin{array}{l}\text { Volatile } \\
\text { Content } \\
\text { (Percent) }\end{array}$ & $\begin{array}{l}\text { Test } \\
\text { Temperature } \\
\left({ }^{\circ} \mathrm{C}\right)\end{array}$ & $\begin{array}{l}\text { Ultimate } \\
\text { Strength } \\
\text { (MPa) }\end{array}$ & $\begin{array}{l}\text { Modulus } \\
(\mathrm{MPa})\end{array}$ & $\begin{array}{l}\text { Deflection } \\
\text { (Percent) }\end{array}$ \\
\hline $6 \mathrm{E}$ & 0.621 & 0.18 & 25 & 10.76 & 841.2 & 1.6 \\
$6 \mathrm{~F}$ & 0.615 & 0.24 & 25 & 16.55 & 1227.3 & 1.8 \\
$6 \mathrm{D}$ & 0.621 & 0.23 & 74 & 6.76 & 689.5 & 1.3 \\
$6 \mathrm{E}$ & 0.618 & 0.19 & 74 & 8.69 & 634.3 & 2.2 \\
$6 \mathrm{~F}$ & 0.613 & 0.20 & 74 & 12.27 & 827.4 & 2.0 \\
\hline
\end{tabular}


The values listed for the tensile and compressive properties were the average of three test specimens. It should be pointed out that there are not any data in Tables 5 through 8 for $0.1 \mathrm{~g} / \mathrm{cm}^{3}$ PSBF. All attempts to mold test specimens from the $0.1 \mathrm{~g} / \mathrm{cm}^{3}$ preexpanded beads failed because of very poor fusion. The $0.1 \mathrm{~g} / \mathrm{cm}^{3}$ test specimens would crumble under slight pressure while being handled. The probable reason for poor fusion is because the beads did not contain enough pentane after preexpansion to produce additional expansion and fusion during the fusion cycle. Therefore, the $0.1 \mathrm{~g} / \mathrm{cm}^{3}$ PSBF was dropped from further investigations.

It also should be noted that there are several groups of $0.6 \mathrm{~g} / \mathrm{cm}^{3}$ PSBF test specimens. When the $0.6 \mathrm{~g} / \mathrm{cm}^{3}$ PSBF test specimens, Groups $A$ and $B$, were placed in a $55^{\circ} \mathrm{C}$ oven for heat aging, approximately one-half of them grew in size, making them unusable. In an attempt to obtain usable $0.6 \mathrm{~g} / \mathrm{cm}^{3}$ heat-aged test specimens, some of the as-received beads were aged at room temperature to reduce the pentane content to a lower level. Four additional groups of $0.6 \mathrm{~g} / \mathrm{cm}^{3}$ test specimens were molded with 2.70 percent volatile content (Groups $6 \mathrm{C}$ and $6 \mathrm{D}$ ) and 3.18 percent volatile content (Groups $6 \mathrm{E}$ and $6 \mathrm{~F}$ ). Only fair fusion was obtained with Group $6 \mathrm{C}$, and only enough test specimens survived handling to obtain as-molded mechanical properties data. The test specimens from Groups $6 \mathrm{D}, 6 \mathrm{E}$, and $6 \mathrm{~F}$ did not grow in size when heat aged in a $55^{\circ} \mathrm{C}$ oven.

An extra group of $0.3 \mathrm{~g} / \mathrm{cm}^{3}$ PSBF test specimens, Group $\mathrm{A}^{-}$, was also molded to verify the low tensile strength values obtained from Group 3A.

Two problems encountered while testing the specimens at $-54^{\circ} \mathrm{C}$ and $74^{\circ} \mathrm{C}$ influenced the test results. During testing of the tensile specimens at $-54^{\circ} \mathrm{C}$, it was noted that excessive amounts of moisture condensed on the test specimens when the test chamber was opened to change test specimens in the fixture. This moisture appeared to have weakened the bond between the test specimen and the adhesive, resulting in failure at that point in most of the specimens. The 0.5 and $0.6 \mathrm{~g} / \mathrm{cm}^{3}$ test specimens actually failed in the bondline between the adhesive and aluminum plug. Therefore, most of the $-54^{\circ} \mathrm{C}$ tensile data was probably lower than it should have been.

The other problem encountered was testing of both the as-molded compressive and tensile test specimen at $74^{\circ} \mathrm{C}$. During the 30 -minute conditioning at $74^{\circ} \mathrm{C}$ before testing, the $0.4,0.5$ and $0.6 \mathrm{~g} / \mathrm{cm}^{3}$ test specimens started to expand and grew out of shape because of an excessive amount of residual pentane in the test specimens. These data were considered to be higher than they should have been because of an increase in the cross-sectional area. 


\section{Effect of Fusion Temperature}

The effects of fusion temperature on the mechanical properties were determined by comparing data from the A Groups to the data from the B Groups listed in Tables 5 through 8 . In most cases, the ultimate tensile strength of the as-molded (Table 5) and heat-aged (Table 6 ) were higher for the test specimens molded at $107^{\circ} \mathrm{C}$ and tested at $-54,25$, and $74^{\circ} \mathrm{C}$. This increase in ultimate tensile strength was more pronounced for the 0.3 and $0.4 \mathrm{~g} / \mathrm{cm}^{3}$ PSBF than for the 0.5 and $0.6 \mathrm{~g} / \mathrm{cm}^{3}$ PSBF. As mentioned above, the data obtained from the 0.5 and $0.6 \mathrm{~g} / \mathrm{cm}^{3}$ test specimen at $107^{\circ} \mathrm{C}$ were questionable because of specimen expansion during the test. The tensile moduli of the $0.3 \mathrm{~g} / \mathrm{cm}^{3}$ as-molded and heat-aged PSBF were higher for the specimen molded at $107^{\circ} \mathrm{C}$ when tested at all three temperatures. This was not the case for the other PSBF densitites, which showed mixed results. Generally, the tensile moduli for the as-molded and heat-aged PSBF with densities of $0.4 \mathrm{~g} / \mathrm{cm}^{3}$ or above appeared to be independent of the fusion temperature.

The tensile elongation for the as-molded and heat-aged specimens showed no significant differences for the tensile elongation of the test specimens molded at 100 and $107^{\circ} \mathrm{C}$.

The compressive strength and modulus (Tables 7 and 8) of all the densities of PSBF appeared to be independent of the fusion temperature at all three test temperatures. In some cases, the higher fusion temperature of $107^{\circ} \mathrm{C}$ resulted in lower values for the compressive strength and modulus. In general, there were no significant differences between the values obtained for these properties from specimens molded at 100 and $107^{\circ} \mathrm{C}$.

Comparison of the deflection at ultimate compression data in Tables 7 and 8 also showed that the compressive deflection was independent of the fusion temperature.

\section{Effect of Residual Pentane Content in PSBF}

The data in Tables 5 through 8 were reduced further by averaging the results from each density of PSBF molded at the two fusion temperatures. These overall average values of the tensile and compressive test specimens are listed in Tables 9 and 10 . The data for the $0.2 \mathrm{~g} / \mathrm{cm}^{3}$ PSBF, reported previously, are included for comparison purposes. The tensile strength and moduli data in Tables 9 and 10 are shown in Figures 1 and 2 as a function of PSBF density for the three test temperatures. In general, the tensile strength and modulus of the PSBF were improved by removal of the residual pentane, especially when tested at $74^{\circ} \mathrm{C}$. This was expected since the pentane dissolved in the polystyrene resin acts as a plasticizer, causing lower mechanical properties. This 
Table 9. Overall Average Tensile Properties of As-Molded (AM) and Heat-Aged (HA) PSBF

\begin{tabular}{|c|c|c|c|c|c|c|c|c|c|}
\hline \multirow{2}{*}{$\begin{array}{l}\text { Nominal } \\
\text { Density } \\
\left(\mathrm{g} / \mathrm{cm}^{3}\right)\end{array}$} & \multicolumn{2}{|c|}{$\begin{array}{l}\text { Volatile } \\
\text { Content } \\
\text { (Percent) }\end{array}$} & \multirow{2}{*}{$\begin{array}{l}\text { Test } \\
\text { Temperature } \\
\left({ }^{\circ} \mathrm{C}\right)\end{array}$} & \multicolumn{2}{|c|}{$\begin{array}{l}\text { Strength } \\
\text { (MPa) }\end{array}$} & \multicolumn{2}{|c|}{$\begin{array}{l}\text { Modulus } \\
\text { (MPa) }\end{array}$} & \multicolumn{2}{|c|}{$\begin{array}{l}\text { Elongation } \\
\text { (Percent) }\end{array}$} \\
\hline & $\mathrm{AM}$ & $\mathrm{HA}$ & & $\mathrm{AM}$ & $\mathrm{HA}$ & $\mathrm{AM}$ & $\mathrm{HA}$ & $\mathrm{AM}$ & $\mathrm{HA}$ \\
\hline 0.2 & $\begin{array}{l}1.4 \\
1.4 \\
1.4\end{array}$ & $\begin{array}{l}<0.2 \\
<0.2 \\
<0.2\end{array}$ & $\begin{array}{r}-54 \\
25 \\
74\end{array}$ & $\begin{array}{l}0.77 \\
0.77 \\
1.03\end{array}$ & $\begin{array}{l}0.51 \\
0.57 \\
0.62\end{array}$ & $\begin{array}{l}221 \\
188 \\
150\end{array}$ & $\begin{array}{l}220 \\
174 \\
127\end{array}$ & $\begin{array}{l}0.35 \\
0.44 \\
0.76\end{array}$ & $\begin{array}{l}0.23 \\
0.23 \\
0.50\end{array}$ \\
\hline 0.3 & $\begin{array}{l}1.4 \\
1.6 \\
1.6\end{array}$ & $\begin{array}{l}0.18 \\
0.19 \\
0.18\end{array}$ & $\begin{array}{r}-54 \\
25 \\
74\end{array}$ & $\begin{array}{l}0.26 \\
0.43 \\
0.51\end{array}$ & $\begin{array}{l}0.35 \\
0.71 \\
0.68\end{array}$ & $\begin{array}{l}211 \\
304 \\
240\end{array}$ & $\begin{array}{l}352 \\
328 \\
307\end{array}$ & $\begin{array}{l}0.16 \\
0.16 \\
0.27\end{array}$ & $\begin{array}{l}0.09 \\
0.22 \\
0.22\end{array}$ \\
\hline 0.4 & $\begin{array}{l}2.4 \\
2.6 \\
2.6\end{array}$ & $\begin{array}{l}0.22 \\
0.22 \\
0.24\end{array}$ & $\begin{array}{r}-54 \\
25 \\
74\end{array}$ & $\begin{array}{l}1.38 \\
2.30 \\
1.64\end{array}$ & $\begin{array}{l}1.67 \\
2.72 \\
2.44\end{array}$ & $\begin{array}{l}566 \\
558 \\
267\end{array}$ & $\begin{array}{l}686 \\
545 \\
493\end{array}$ & $\begin{array}{l}0.26 \\
0.43 \\
1.09\end{array}$ & $\begin{array}{l}0.25 \\
0.52 \\
0.52\end{array}$ \\
\hline 0.5 & $\begin{array}{l}2.8 \\
2.8 \\
2.6\end{array}$ & $\begin{array}{l}0.54 \\
0.52 \\
0.47\end{array}$ & $\begin{array}{r}-54 \\
25 \\
74\end{array}$ & $\begin{array}{l}2.26 \\
3.92 \\
1.10\end{array}$ & $\begin{array}{l}2.23 \\
3.80 \\
3.20\end{array}$ & $\begin{array}{l}828 \\
762 \\
160\end{array}$ & $\begin{array}{r}848 \\
1300 \\
1390\end{array}$ & $\begin{array}{l}0.28 \\
0.52 \\
1.96\end{array}$ & $\begin{array}{l}0.26 \\
0.52 \\
0.52\end{array}$ \\
\hline $0.6 *$ & $\begin{array}{l}3.7 \\
3.8 \\
3.6\end{array}$ & $\begin{array}{l}x+70 \\
1.70 \\
i x\end{array}$ & $\begin{array}{r}-54 \\
25 \\
74\end{array}$ & $\begin{array}{l}1.64 \\
5.29 \\
0.04\end{array}$ & $\underset{t x}{6.93}$ & $\begin{array}{r}1125 \\
669 \\
58\end{array}$ & $\begin{array}{r}\text { tht } \\
893 \\
t x\end{array}$ & $\begin{array}{l}0.16 \\
1.12 \\
1.38\end{array}$ & 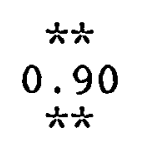 \\
\hline
\end{tabular}

*Average data from only Group A and Group B.

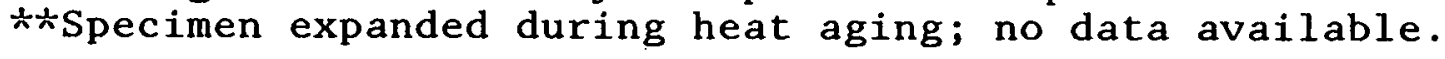


Table 10. Overall Average Compressive Properties of As-Molded (AM) and Heat-Aged (HA) PSBF

\begin{tabular}{|c|c|c|c|c|c|c|c|c|c|}
\hline \multirow{2}{*}{$\begin{array}{l}\text { Nominal } \\
\text { Density } \\
\left(\mathrm{g} / \mathrm{cm}^{3}\right)\end{array}$} & \multicolumn{2}{|c|}{$\begin{array}{l}\text { Volatile } \\
\text { Content } \\
\text { (Percent) }\end{array}$} & \multirow{2}{*}{$\begin{array}{l}\text { Test } \\
\text { Temperature } \\
\left({ }^{\circ} \mathrm{C}\right)\end{array}$} & \multicolumn{2}{|c|}{$\begin{array}{l}\text { Strength } \\
(\mathrm{MPa})\end{array}$} & \multicolumn{2}{|c|}{$\begin{array}{l}\text { Modulus } \\
(\mathrm{MPa})\end{array}$} & \multicolumn{2}{|c|}{$\begin{array}{l}\text { Deflection } \\
\text { (Percent) }\end{array}$} \\
\hline & $\mathrm{AM}$ & HA & & $\mathrm{AM}$ & $\mathrm{HA}$ & $\mathrm{AM}$ & $\mathrm{HA}$ & $\mathrm{AM}$ & HA \\
\hline 0.2 & $\begin{array}{l}1.4 \\
1.4 \\
1.4\end{array}$ & $\begin{array}{l}<0.2 \\
<0.2 \\
<0.2\end{array}$ & $\begin{array}{r}-54 \\
25 \\
74\end{array}$ & $\begin{array}{l}4.32 \\
2.78 \\
1.70\end{array}$ & $\begin{array}{l}3.81 \\
2.83 \\
2.05\end{array}$ & $\begin{array}{l}186 \\
152 \\
128\end{array}$ & $\begin{array}{l}161 \\
146 \\
127\end{array}$ & $\begin{array}{l}4.0 \\
3.2 \\
2.5\end{array}$ & $\begin{array}{l}4.0 \\
3.2 \\
2.5\end{array}$ \\
\hline 0.3 & $\begin{array}{l}1.4 \\
1.6 \\
1.6\end{array}$ & $\begin{array}{l}0.18 \\
0.19 \\
0.18\end{array}$ & $\begin{array}{r}-54 \\
25 \\
74\end{array}$ & $\begin{array}{l}5.89 \\
4.09 \\
2.43\end{array}$ & $\begin{array}{l}5.76 \\
4.74 \\
3.34\end{array}$ & $\begin{array}{l}326 \\
280 \\
140\end{array}$ & $\begin{array}{l}380 \\
303 \\
266\end{array}$ & $\begin{array}{l}2.4 \\
2.8 \\
2.4\end{array}$ & $\begin{array}{l}2.8 \\
2.9 \\
2.4\end{array}$ \\
\hline 0.4 & $\begin{array}{l}2.4 \\
2.6 \\
2.6\end{array}$ & $\begin{array}{l}0.22 \\
0.22 \\
0.24\end{array}$ & $\begin{array}{r}-54 \\
25 \\
74\end{array}$ & $\begin{array}{r}13.06 \\
8.07 \\
2.08\end{array}$ & $\begin{array}{r}14.00 \\
10.96 \\
7.33\end{array}$ & $\begin{array}{l}448 \\
414 \\
105\end{array}$ & $\begin{array}{l}502 \\
502 \\
424\end{array}$ & $\begin{array}{l}4.3 \\
3.6 \\
2.8\end{array}$ & $\begin{array}{l}3.8 \\
3.6 \\
3.2\end{array}$ \\
\hline 0.5 & $\begin{array}{l}2.8 \\
2.8 \\
2.6\end{array}$ & $\begin{array}{l}0.54 \\
0.52 \\
0.47\end{array}$ & $\begin{array}{r}-54 \\
25 \\
74\end{array}$ & $\begin{array}{r}21.16 \\
12.95 \\
0.73\end{array}$ & $\begin{array}{l}21.79 \\
16.17 \\
10.14\end{array}$ & $\begin{array}{r}531 \\
662 \\
10\end{array}$ & $\begin{array}{l}869 \\
707 \\
527\end{array}$ & $\begin{array}{l}5.0 \\
3.9 \\
--\end{array}$ & $\begin{array}{l}3.4 \\
3.5 \\
2.8\end{array}$ \\
\hline $0.6 *$ & $\begin{array}{l}3.7 \\
3.8 \\
3.6\end{array}$ & $\underbrace{+70}_{t 大}$ & $\begin{array}{r}-54 \\
25 \\
74\end{array}$ & $\begin{array}{r}29.26 \\
15.10 \\
0.02\end{array}$ & $\begin{array}{l}\frac{t}{x} \\
19.70 \\
\frac{x}{x}\end{array}$ & $\begin{array}{l}982 \\
682 \\
--\end{array}$ & 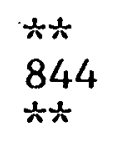 & $\begin{array}{l}4.7 \\
3.8 \\
--\end{array}$ & $\begin{array}{l}\frac{x}{x} \\
4.6 \\
x+6\end{array}$ \\
\hline
\end{tabular}




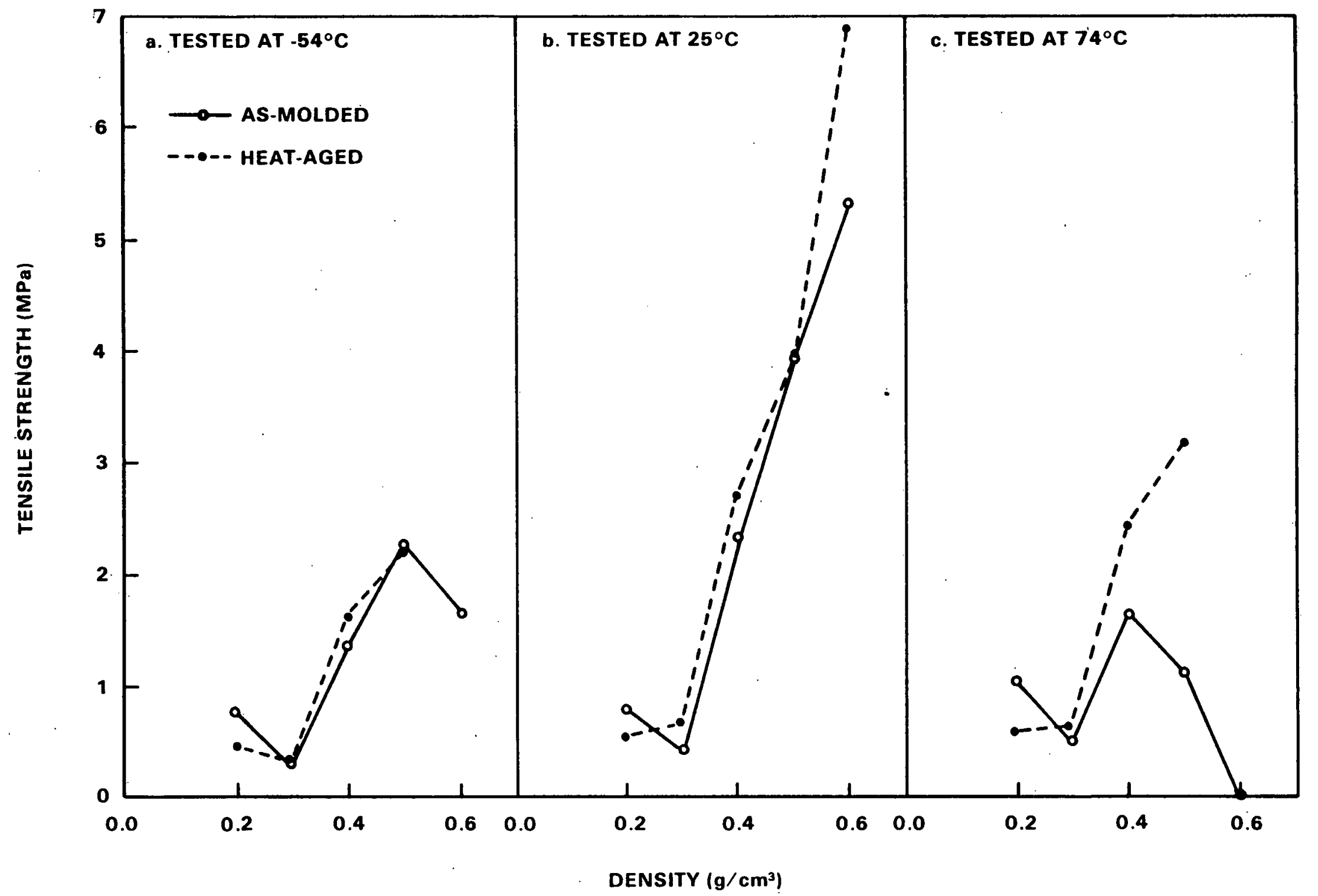

Figure 1. Tensile Strength Versus Density of PSBF

N 


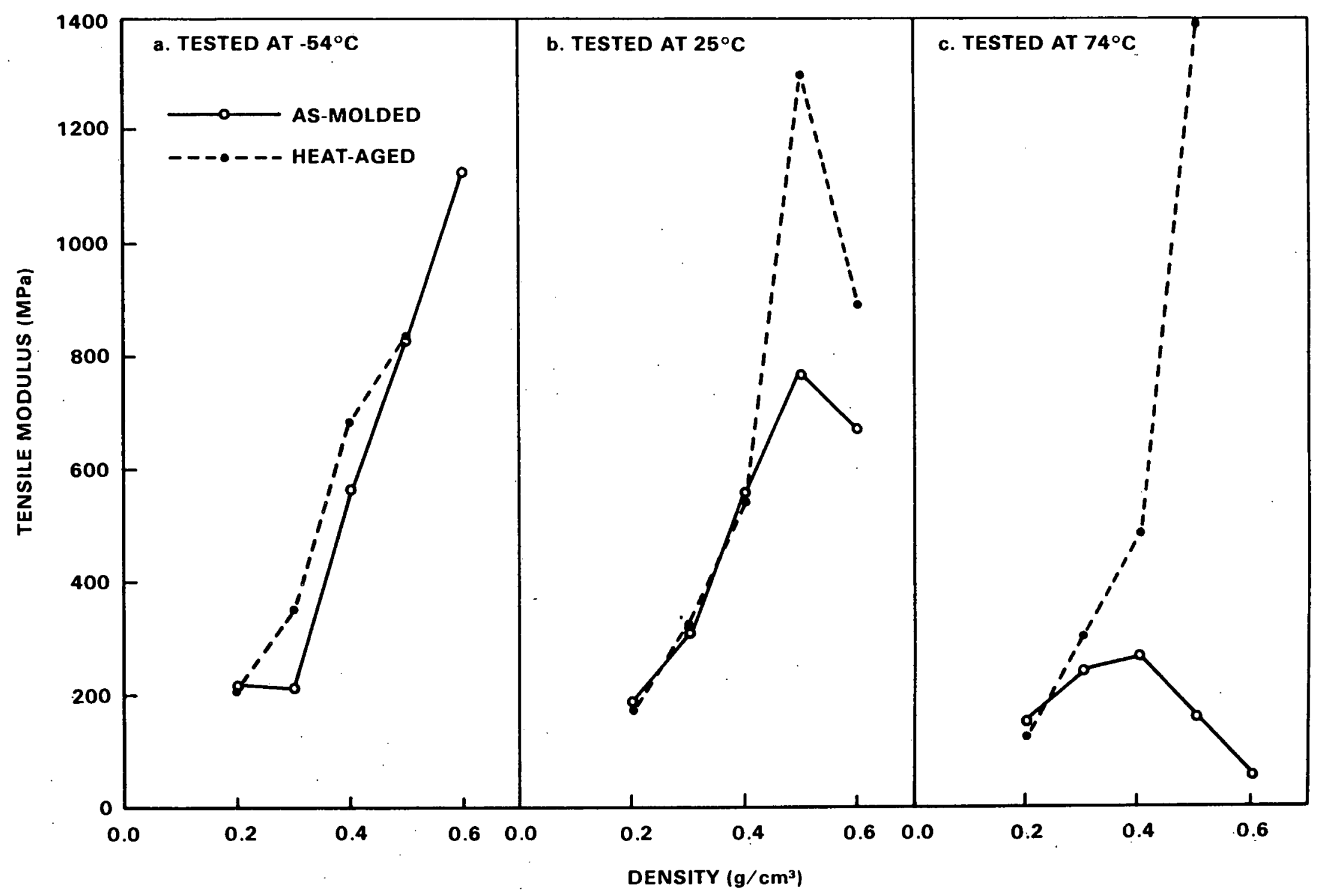

Figure 2. Tensile Modulus Versus Density of PSBF 
was especially true for the 0.5 and $0.6 \mathrm{~g} / \mathrm{cm}^{3}$ PSBF when tested at $74^{\circ} \mathrm{C}$. The high level of residual pentane ( 2.5 percent) drastically lowered the glass transition temperature to some temperature below $74^{\circ} \mathrm{C}$, resulting in expansion of the test specimen during testing. The tensile modulus value for the heat-aged $0.6 \mathrm{~g} / \mathrm{cm}^{3}$ at $25^{\circ} \mathrm{C}$ may be attributed to the 1.70 percent residual pentane content. The tensile elongation data indicated that this property did not change significantly when the residual pentane was reduced, except for the 0.5 and $0.6 \mathrm{~g} / \mathrm{cm}^{3}$ PSBF tested at $74^{\circ} \mathrm{C}$.

The compressive strength data for the as-molded and heat-aged test specimens are shown in Figure 3. Removal of the residual pentane significantly increased the compressive strengths of the $0.4 \mathrm{~g} / \mathrm{cm}^{3}$ and higher densities of PSBF when tested at 25 and $74^{\circ} \mathrm{C}$. The plasticizing effect of the pentane dissolved in the PSBF was minimal at $-54^{\circ} \mathrm{C}$.

The compressive modulus data for the as-molded and heat-aged test specimens are shown in Figure 4. This figure shows an increase in the compressive modulus, which is attributed to the removal of the pentane from PSBF densities above $0.3 \mathrm{~g} / \mathrm{cm}^{3}$. The most.significant increases occurred at the $74^{\circ} \mathrm{C}$ test temperature.

The compressive deflection was not affected by removal of the pentane from the $0.2,0.3$ and $0.4 \mathrm{~g} / \mathrm{cm}^{3}$ PSBF. However, the compressive deflection was significantly lower for the heat-aged $0.5 \mathrm{~g} / \mathrm{cm}^{3}$ PSBF when tested at $-54^{\circ} \mathrm{C}$ and higher when tested at $74^{\circ} \mathrm{C}$.

\section{Effect of Test Temperature}

The effect of the test temperature on the mechanical properties can be determined by comparing the data shown in the three sections of Figures 1 through 4 for a given density of PSBF.

The tensile strength (Figure 1 ) of the 0.2 and $0.3 \mathrm{~g} / \mathrm{cm}^{3}$ did not change drastically over the temperature range of -54 to $74^{\circ} \mathrm{C}$. The PSBF densities higher than $0.3 \mathrm{~g} / \mathrm{cm}^{3}$ increased significantly from -54 to $25^{\circ} \mathrm{C}$ and decreased sharply between 25 and $74^{\circ} \mathrm{C}$. The tensile values of the PSBF tested at $-54^{\circ} \mathrm{C}$ may be lower than expected because of the moisture condensation of test specimens, as previously mentioned.

In general, the tensile modulus (Figure 2) decreased as the temperature increased from -54 to $74^{\circ} \mathrm{C}$, with a more significant decrease as the density increased. The one exception to this trend was the heat-aged $0.5 \mathrm{~g} / \mathrm{cm}^{3} \mathrm{PSBF}$, which showed an increase in tensile modulus as the temperature increased. The tensile elongation values showed a slight increase for each density as the test temperature increased. The magnitude of the increase in elongation was greater for the higher densities of PSBF. 


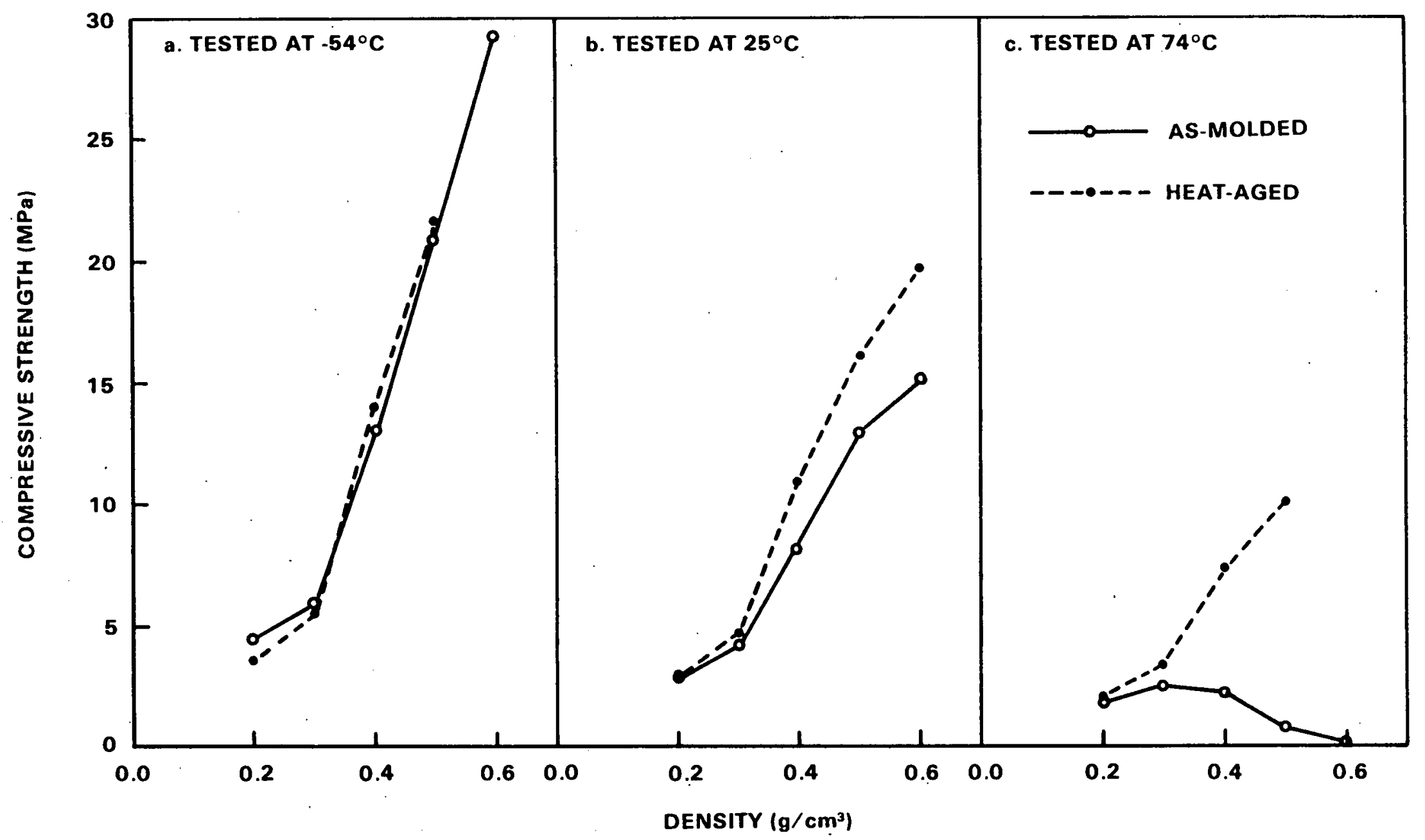

Figure 3. Compressive Strength Versus Density of PSBF 


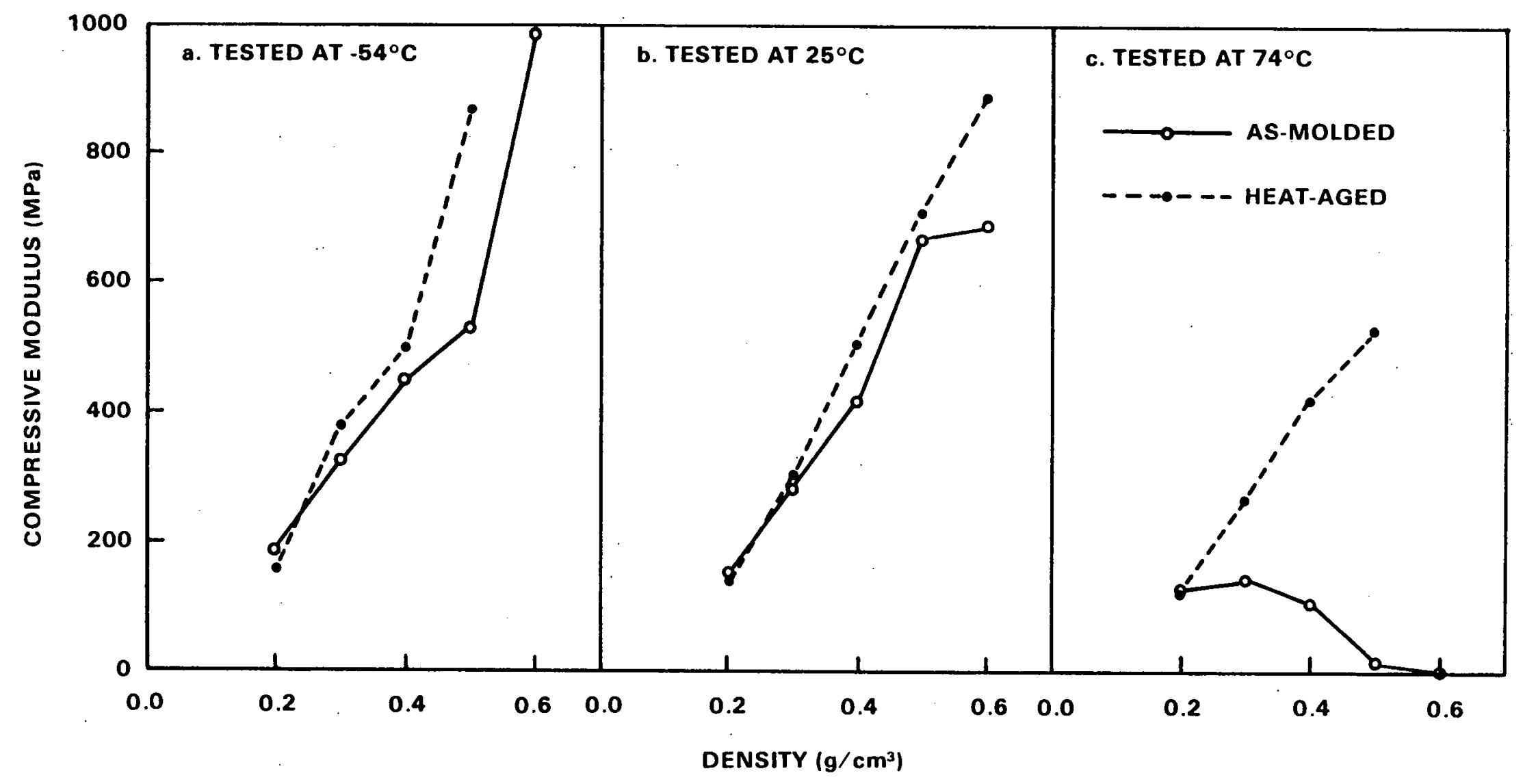

Figure 4. Compressive Modulus Versus Density of PSBF 
The compressive strength (Figure 3), compressive modulus (Figure 4), and compressive deflection decreased in values as the temperature increased from -54 to $74^{\circ} \mathrm{C}$, with greater decrease in values as the density increased. This behavior is typical of thermoplastic materials such as polystyrene.

\section{Effect of PSBF Density}

Using the data shown in Figures 1 through 4, the effect of PSBF density on the tensile and compressive properties were determined. In general, the tensile and compressive properties increased as the density of the PSBF increased, with a few exceptions. The tensile strength of the $0.3 \mathrm{~g} / \mathrm{cm}^{3}$ PSBF was lower than the data previously reported for the $0.2 \mathrm{~g} / \mathrm{cm}^{3}$ PSBF. This abnormality may be explained by the possibility that the $0.3 \mathrm{~g} / \mathrm{cm}^{3}$ preexpanded beads had a lesser amount of pentane at the time of molding than did the $0.2 \mathrm{~g} / \mathrm{cm}^{3}$ preexpanded beads. The tensile strength obtained for PSBF was found to be dependent on the pentane content of the preexpanded beads at the time they were molded.

Another exception was the tensile modulus of the as-molded and heat-aged $0.6 \mathrm{~g} / \mathrm{cm}^{3}$ PSBF, which was lower than the corresponding data for the $0.5 \mathrm{~g} / \mathrm{cm}^{3}$ PSBF. These low values for the $0.6 \mathrm{~g} / \mathrm{cm}^{3}$ PSBF were attributed to the fact that the $0.6 \mathrm{~g} / \mathrm{cm}^{3}$ PSBF had a higher residual pentane content. The higher the residual pentane content, the greater the plasticizing effect it has on the PSBF. This plasticizing effect became more pronounced as the temperature was increased, as was seen with the drastic drop in mechanical properties of the $0.4 \mathrm{~g} / \mathrm{cm}^{3}$ and higher densities of PSBF when tested at $74^{\circ} \mathrm{C}$.

\section{Effect of Initial Pentane Content of EPS Beads}

As mentioned, when the $0.6 \mathrm{~g} / \mathrm{cm}^{3}$ test specimens were placed in a $55^{\circ} \mathrm{C}$ oven for residual pentane removal, approximately one-half of the test specimens were distorted by further expansion of the PSBF. This expansion was caused by the high residual pentane content $\left(\sim 3.7\right.$ percent) in the $0.6 \mathrm{~g} / \mathrm{cm}^{3} \mathrm{PSBF}$. The residual pentane in effect reduced the temperature at which the polystyrene resin softens enough to allow the expansion of the pentane gas. This expansion of the PSBF as-molded test specimens with densities of $0.4 \mathrm{~g} / \mathrm{cm}^{3}$ and higher also occurred when they were tested at $74^{\circ} \mathrm{C}$, as previously mentioned. This expansion of the higher-density PSBF could present a problem for some applications.

In order to solve this problem, some as-received beads were aged at room temperature to lower the pentane content from 6.0 to 3.2 and 2.7 percent. Four additional sets of $0.6 \mathrm{~g} / \mathrm{cm}^{3}$ PSBF test 
specimens were molded from the beads with the low level of pentane content. One set from each pentane level was molded at a $100^{\circ} \mathrm{C}$ fusion temperature, and one set was molded using a $107^{\circ} \mathrm{C}$ fusion temperature. The test data from these test specimens are listed in Tables 11 and 12 along with the data from the original $0.6 \mathrm{~g} / \mathrm{cm}^{3}$ PSBF for comparison purposes.

The test specimens from these four groups did not distort or expand when placed in a $55^{\circ} \mathrm{C}$ oven for pentane removal. In addition, they did not expand at the $74^{\circ} \mathrm{C}$ test temperature.

From the test data in Tables 11 and 12 , it was apparent that the beads containing 2.7 percent pentane and molded at $100^{\circ} \mathrm{C}$ did not produce a foam with adequate fusion. The 2.7 percent pentane beads molded at $107^{\circ} \mathrm{C}$ (Group 6D) resulted in a higher degree of fusion, but was still marginal because the tensile specimen broke during handling at $-54^{\circ} \mathrm{C}$.

The test specimens molded at $100^{\circ} \mathrm{C}$ with initial pentane content of 3.2 percent (Group 6E) had mechanical properties very similar to Group $6 \mathrm{C}$, which were molded at $107^{\circ} \mathrm{C}$ using beads with 2.7 percent pentane. Group $6 \mathrm{~F}$ test specimen, molded at $107^{\circ} \mathrm{C}$ with 3.2 percent pentane, had acceptable fusion and mechanical properties.

It was concluded from the data in Tables 11 and 12 that $0.6 \mathrm{~g} / \mathrm{cm}^{3}$ PSBF can be successfully molded to withstand a temperature of $74^{\circ} \mathrm{C}$ by using beads with a lower pentane content ( 3.2 percent minimum) and a higher fusion temperature $\left(107^{\circ} \mathrm{C}\right)$. This same conclusion could probably be made for the 0.4 and $0.5 \mathrm{~g} / \mathrm{cm}^{3}$ PSBF; however, an attempt to verify it for these densities of PSBF was not undertaken.

\section{ACCOMPLISHMENTS}

The tensile and compressive properties of PSBF were characterized as a function of density, 0.3 to $0.6 \mathrm{~g} / \mathrm{cm}^{3}$, at three test temperatures, $-54,25$, and $74^{\circ} \mathrm{C}$. Results of this study determined that $0.1 \mathrm{~g} / \mathrm{cm}^{3}$ PSBF made from BF-414 cannot be used as an encapsulant because of poor bead fusion. The maximum use temperature of PSBF with $0.4 \mathrm{~g} / \mathrm{cm}^{3}$ and higher densities can be increased by starting with beads containing lower amounts of pentane and increasing the fusion temperature. 
Table 11. Tensile Properties of $0.6 \mathrm{~g} / \mathrm{cm}^{3}$ As-Molded (AM) and Heat-Aged (HA) PSBF With Different Initial Volatile Contents

\begin{tabular}{|c|c|c|c|c|c|c|c|c|c|}
\hline \multirow{2}{*}{$\begin{array}{l}\text { Test } \\
\text { Group }\end{array}$} & \multirow{2}{*}{$\begin{array}{l}\text { Initial } \\
\text { Volatile } \\
\text { Content } \\
\text { (Percent) }\end{array}$} & \multicolumn{2}{|c|}{ Temperature $\left({ }^{\circ} \mathrm{C}\right)$} & \multicolumn{2}{|c|}{$\begin{array}{l}\text { Strength } \\
\text { (MPa) }\end{array}$} & \multicolumn{2}{|c|}{$\begin{array}{l}\text { Modulus } \\
\text { (MPa) }\end{array}$} & \multicolumn{2}{|c|}{$\begin{array}{l}\text { Elongation } \\
(\mathrm{MPa})\end{array}$} \\
\hline & & Fusion & Test & AM & $\mathrm{HA}$ & AM & $\mathrm{HA}$ & AM & $\mathrm{HA}$ \\
\hline $6 \mathrm{~A}$ & 6.0 & 100 & $\begin{array}{r}-54 \\
25 \\
74\end{array}$ & $\begin{array}{l}1.86 \\
5.41 \\
0.03\end{array}$ & $\begin{array}{l}* \\
7.05 \\
*\end{array}$ & $\begin{array}{r}1090 \\
669 \\
34\end{array}$ & $\begin{array}{l}- \\
-\end{array}$ & $\begin{array}{l}0.15 \\
1.11 \\
1.63\end{array}$ & $\begin{array}{l}- \\
0.94\end{array}$ \\
\hline $6 \mathrm{~B}$ & 6.0 & 107 & $\begin{array}{r}-54 \\
25 \\
74\end{array}$ & $\begin{array}{l}1.41 \\
5.17 \\
0.05\end{array}$ & $\begin{array}{l}t \\
6.81 \\
\vdots\end{array}$ & $\begin{array}{r}1160 \\
669 \\
83\end{array}$ & -910 & $\begin{array}{l}0.18 \\
1.12 \\
1.02\end{array}$ & $\begin{array}{l}- \\
0.87 \\
-\end{array}$ \\
\hline $6 \mathrm{C}$ & 2.7 & 100 & $\begin{array}{r}-54 \\
25 \\
74\end{array}$ & $\begin{array}{l}x \\
0.21 \\
x\end{array}$ & $\begin{array}{l}x+2 \\
-\end{array}$ & $\begin{array}{l}- \\
- \\
-\end{array}$ & $\begin{array}{l}- \\
-\end{array}$ & $\begin{array}{l}- \\
0.03 \\
-\end{array}$ & $\begin{array}{l}- \\
-\end{array}$ \\
\hline 6D & 2.7 & 107 & $\begin{array}{r}-54 \\
25 \\
74\end{array}$ & $\begin{array}{l}\text { 낭 } \\
0.85 \\
0.18\end{array}$ & $\begin{array}{l}0.10 \\
1.38 \\
0.81\end{array}$ & $\begin{array}{r}- \\
944 \\
575\end{array}$ & $\begin{array}{r}882 \\
1151 \\
889\end{array}$ & $\begin{array}{l}- \\
0.27 \\
0.11\end{array}$ & $\begin{array}{l}0.07 \\
0.13 \\
0.11\end{array}$ \\
\hline $6 \mathrm{E}$ & 3.2 & 100 & $\begin{array}{r}-54 \\
25 \\
74\end{array}$ & $\begin{array}{l}t+d \\
1.48 \\
0.17\end{array}$ & $\begin{array}{l}0.36 \\
1.21 \\
0.95\end{array}$ & $\begin{array}{r}- \\
1129 \\
190\end{array}$ & $\begin{array}{r}1255 \\
1034 \\
724\end{array}$ & $\begin{array}{l}- \\
0.16 \\
0.15\end{array}$ & $\begin{array}{l}0.03 \\
0.11 \\
0.14\end{array}$ \\
\hline $6 F$ & 3.2 & 107 & $\begin{array}{r}-54 \\
25 \\
74\end{array}$ & $\begin{array}{l}0.77 \\
3.00 \\
2.30\end{array}$ & $\begin{array}{l}0.98 \\
2.34 \\
1.79\end{array}$ & $\begin{array}{r}1080 \\
1080 \\
620\end{array}$ & $\begin{array}{r}1214 \\
1262 \\
889\end{array}$ & $\begin{array}{l}0.11 \\
0.28 \\
0.50\end{array}$ & $\begin{array}{l}0.11 \\
0.19 \\
0.27\end{array}$ \\
\hline
\end{tabular}

*Test specimen expanded during heat aging; not able to test.

*-Test specimen broke in handling before testing.

w-:Poor fusion, not enough specimen to heat age. 
Table 12. Compressive Properties of $0.6 \mathrm{~g} / \mathrm{cm}^{3}$ As-Molded (AM) and Heat-Aged (HA) PSBF With Different Initial Volatile Contents

\begin{tabular}{|c|c|c|c|c|c|c|c|c|c|}
\hline \multirow{2}{*}{$\begin{array}{l}\text { Test } \\
\text { Group }\end{array}$} & \multirow{2}{*}{$\begin{array}{l}\text { Initial } \\
\text { Volatile } \\
\text { Content } \\
\text { (Percent) }\end{array}$} & \multicolumn{2}{|c|}{ Temperature $\left({ }^{\circ} \mathrm{C}\right)$} & \multicolumn{2}{|c|}{$\begin{array}{l}\text { Strength } \\
\text { (MPa) }\end{array}$} & \multicolumn{2}{|c|}{$\begin{array}{l}\text { Modulus } \\
(\mathrm{MPa})\end{array}$} & \multicolumn{2}{|c|}{$\begin{array}{l}\text { Deflection } \\
\text { (MPa) }\end{array}$} \\
\hline & & Fusion & Test & AM & $\mathrm{HA}$ & $\mathrm{AM}$ & HA & AM & HA \\
\hline $6 A$ & 6.0 & 100 & $\begin{array}{r}-54 \\
25 \\
74\end{array}$ & $\begin{array}{r}29.92 \\
15.65 \\
0.03\end{array}$ & $\begin{array}{l}* \\
*\end{array}$ & $\begin{array}{r}1034 \\
717 \\
-\quad\end{array}$ & - & $\begin{array}{l}4.2 \\
3.9 \\
-\end{array}$ & $\begin{array}{l}- \\
4.4\end{array}$ \\
\hline $6 \mathrm{~B}$ & 6.0 & 107 & $\begin{array}{r}-54 \\
25 \\
74\end{array}$ & $\begin{array}{r}28.61 \\
14.54 \\
0.02\end{array}$ & $\stackrel{\hbar}{18.72}$ & $\begin{array}{r}931 \\
648 \\
-\end{array}$ & $\begin{array}{l}- \\
-\end{array}$ & $\begin{array}{l}5.2 \\
3.8 \\
-\end{array}$ & $\begin{array}{l}- \\
4.9 \\
-\end{array}$ \\
\hline $6 C$ & 2.7 & 100 & $\begin{array}{r}-54 \\
25 \\
74\end{array}$ & $\begin{array}{l}0.50 \\
1.69 \\
0.68\end{array}$ & $\begin{array}{l}x+5 \\
-\end{array}$ & $\begin{array}{r}28 \\
607 \\
138\end{array}$ & $\begin{array}{l}- \\
- \\
-\end{array}$ & $\begin{array}{l}1.3 \\
0.2 \\
0.9\end{array}$ & $\begin{array}{l}- \\
-\end{array}$ \\
\hline $6 \mathrm{D}$ & 2.7 & 107 & $\begin{array}{r}-54 \\
25 \\
74\end{array}$ & $\begin{array}{l}4.77 \\
6.09 \\
3.08\end{array}$ & $\begin{array}{r}11.31 \\
5.32 \\
6.76\end{array}$ & $\begin{array}{l}400 \\
738 \\
407\end{array}$ & $\begin{array}{l}827 \\
765 \\
690\end{array}$ & $\begin{array}{l}2.1 \\
0.9 \\
2.1\end{array}$ & $\begin{array}{l}1.4 \\
0.8 \\
1.3\end{array}$ \\
\hline $6 \mathrm{E}$ & 3.2 & 100 & $\begin{array}{r}-54 \\
25 \\
74\end{array}$ & $\begin{array}{l}4.77 \\
6.69 \\
3.57\end{array}$ & $\begin{array}{r}14.31 \\
10.76 \\
8.69\end{array}$ & $\begin{array}{l}510 \\
786 \\
186\end{array}$ & $\begin{array}{r}1034 \\
841 \\
634\end{array}$ & $\begin{array}{l}2.1 \\
0.9 \\
3.4\end{array}$ & $\begin{array}{l}1.4 \\
1.6 \\
2.2\end{array}$ \\
\hline $6 \mathrm{~F}$ & 3.2 & 107 & $\begin{array}{r}-54 \\
25 \\
74\end{array}$ & $\begin{array}{r}18.47 \\
15.38 \\
4.96\end{array}$ & $\begin{array}{l}19.99 \\
16.55 \\
12.27\end{array}$ & $\begin{array}{l}745 \\
986 \\
200\end{array}$ & $\begin{array}{r}1151 \\
1227 \\
827\end{array}$ & $\begin{array}{l}2.2 \\
1.6 \\
3.0\end{array}$ & $\begin{array}{l}2.0 \\
1.8 \\
2.0\end{array}$ \\
\hline
\end{tabular}

‘Test specimen expanded during heat aging; not able to test. **Poor fusion, not enough specimen to heat age. 


\section{REFERENCES}

${ }^{1}$ D. J. Fossey, Design Manual for Polystyrene Foam, Relating Physical, Mechanical, and Chemical Properties (Topical Report). Bendix Kansas City: BDX-6 $\overline{13-1033, ~ F e b r u a r y ~} 1975$ (Available from NTIS).

${ }^{2}$ R. C. Swoboda, Feasibility Study of Production of $0.24 \mathrm{~g} / \mathrm{cm}^{3}-$ Density Polystyrene Bead Foam (Topical Report). Bendix Kansas

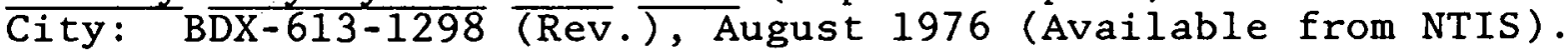

${ }^{3}$ R. C. Swoboda, Feasibility Study for Producing High-Density Polystyrene Bead Foam (Final Report). Bendix Kansas City: BDX-613-1422 (Rev.), January 1976 (Available from NTIS).

${ }^{4} \mathrm{D}$. J. Fossey, Mechanical Properties of $0.2 \mathrm{~g} / \mathrm{cm}^{3}$ Polystyrene Bead Foam (Topical Report). Bendix Kansas City: BDX-613-1725 (Rev. ), January 1978 (Available from NTIS). 
BDX -613-2412

MECHANICAL PROPERTIES OF POLYSTYRENE BEAD ENCAPSULATION FOAM, D. J. Fossey, Topical, March 1980.

The tensile and compressive properties of polystyrene bead foam used as an encapsulant for electronic devices were determined for densities ranging from 0.3 to $0.6 \mathrm{~g} / \mathrm{cm}^{3}$. Data were generated for these properties at $-54,25$, and $74^{\circ} \mathrm{C}$. Data previously reported for $0.2 \mathrm{~g} / \mathrm{cm}^{3}$ are included.

ELECTRICAL: Encapsulants

MECHANICAL PROPERTIES OF POLYSTYRENE BEAD ENCAPSULATION FOAM, D. J. Fossey, Topical, BDX-613-2412, March 1980.

The tensile and compressive properties of polystyrene bead foam used as an encapsulant for electronic devices were determined for densities ranging from 0.3 to $0.6 \mathrm{~g} / \mathrm{cm}^{3}$. Data were generated for these properties at $-54,25$, and $74^{\circ} \mathrm{C}$. Data previously reported for $0.2 \mathrm{~g} / \mathrm{cm}^{3}$ are included.

MECHANICAL PROPERTIES OF POLYSTYRENE BEAD ENCAPSULATION FOAM, D. J. Fossey, Topical, BDX-613-2412, March 1980.

The tensile and compressive properties of polystyrene bead foam used as an encapsulant for electronic devices were determined for densities ranging from 0.3 to $0.6 \mathrm{~g} / \mathrm{cm}^{3}$. Data were generated for these properties at $-54,25$, and $74^{\circ} \mathrm{C}$. Data previously reported for $0.2 \mathrm{~g} / \mathrm{cm}^{3}$ are included. 